\title{
1 Uncovering novel mutational signatures by de novo extraction with
}

2 SigProfilerExtractor

3 S M Ashiqul Islam ${ }^{1,2,3}$, Yang $\mathrm{Wu}^{4}$, Marcos Díaz-Gay ${ }^{1,2,3}$, Erik N Bergstrom ${ }^{1,2,3}$, Yudou He ${ }^{1,2,3}$, 4 Mark Barnes $^{1,2,3}$, Mike Vella ${ }^{5}$, Jingwei Wang ${ }^{6}$, Jon W Teague ${ }^{6}$, Peter Clapham ${ }^{6}$, Sarah Moody ${ }^{6}$, 5 Sergey Senkin ${ }^{7}$, Yun Rose $\mathrm{Li}^{8}$, Laura Riva ${ }^{6}$, Tongwu Zhang ${ }^{9}$, Andreas J Gruber ${ }^{10,11}$, Raviteja 6 Vangara $^{12}$, Christopher D Steele ${ }^{13}$, Burçak Otlu ${ }^{1,2,3}$, Azhar Khandekar ${ }^{1,2,3}$, Ammal Abbasi ${ }^{1,2,3}$, 7 Laura Humphreys ${ }^{6}$, Natalia Syulyukina ${ }^{2}$, Samuel W Brady ${ }^{14}$, Boian S Alexandrov ${ }^{12}$, Nischalan

8 Pillay $^{13,15}$, Jinghui Zhang ${ }^{14}$, David J Adams ${ }^{6}$, Iñigo Martincorena ${ }^{6}$, David C Wedge ${ }^{10,11}$, Maria

9 Teresa Landi ${ }^{9}$, Paul Brennan ${ }^{7}$, Michael R Stratton ${ }^{6}$, Steven G Rozen ${ }^{4}$, and Ludmil B

10 Alexandrov $1,2,3^{*}$

\section{Affiliations}

$14{ }^{1}$ Department of Cellular and Molecular Medicine, UC San Diego, La Jolla, CA, 92093, USA

15 2Department of Bioengineering, UC San Diego, La Jolla, CA, 92093, USA

$16{ }^{3}$ Moores Cancer Center, UC San Diego, La Jolla, CA, 92037, USA

$17{ }^{4}$ Centre for Computational Biology and Programme in Cancer \& Stem Cell Biology, Duke NUS

18 Medical School, 169857, Singapore

$19{ }^{5}$ NVIDIA Corporation, 2788 San Tomas Expressway, Santa Clara, CA, 95051, USA

${ }^{6}$ Cancer, Ageing and Somatic Mutation, Wellcome Sanger Institute, Wellcome Genome Campus, Cambridge, CB10 1SA, UK

${ }^{7}$ Genetic Epidemiology Group, International Agency for Research on Cancer, 69372 Lyon CEDEX 08, France

${ }^{8}$ Helen Diller Family Comprehensive Cancer Center, San Francisco, CA, 94158, USA

${ }^{9}$ Division of Cancer Epidemiology and Genetics, National Cancer Institute, Bethesda, MD, 20892, USA

${ }^{10}$ Big Data Institute, Nuffield Department of Medicine, University of Oxford, Oxford, OX3 7LF, UK

${ }^{11}$ Manchester Cancer Research Centre, The University of Manchester, Manchester, M20 4GJ, UK

${ }^{12}$ Theoretical Division, Los Alamos National Laboratory, Los Alamos, NM, 87545, USA

${ }^{13}$ Research Department of Pathology, Cancer Institute, University College London, London, WC1E 6BT, UK

${ }^{14}$ Department of Computational Biology, St. Jude Children's Research Hospital, Memphis, 38105, Tennessee, USA

${ }^{15}$ Department of Cellular and Molecular Pathology, Royal National Orthopaedic Hospital NHS Trust, Stanmore, Middlesex, HA7 4LP, UK

*Correspondence should be addressed to L2alexandrov@health.ucsd.edu. 


\section{ABSTRACT}

41 Mutational signature analysis is commonly performed in genomic studies surveying cancer and

42 normal somatic tissues. Here we present SigProfilerExtractor, an automated tool for accurate de

43 novo extraction of mutational signatures for all types of somatic mutations. Benchmarking with a

44 total of 33 distinct scenarios encompassing 1,900 simulated signatures operative in more than

4560,000 unique synthetic genomes demonstrates that SigProfilerExtractor outperforms thirteen

46 other tools across all datasets with and without noise. For simulations with 5\% noise, reflecting

47 high-quality genomic datasets, SigProfilerExtractor outperforms other approaches by elucidating

48 between $20 \%$ and $50 \%$ more true positive signatures while yielding more than 5 -fold less false

49 positive signatures. Applying SigProfilerExtractor to 2,778 whole-genome sequenced cancers

50 reveals three previously missed mutational signatures. Two of the signatures are confirmed in

51 independent cohorts with one of these signatures associating with tobacco smoking. In summary,

52 this report provides a reference tool for analysis of mutational signatures, a comprehensive

53 benchmarking of bioinformatics tools for extracting mutational signatures, and several novel

54 mutational signatures including a signature putatively attributed to direct tobacco smoking

55 mutagenesis in bladder cancer and in normal bladder epithelium. 


\section{INTRODUCTION}

58 De novo extraction of mutational signatures ${ }^{1}$ is an unsupervised machine learning approach

59 where a matrix, $\boldsymbol{M}$, which corresponds to the somatic mutations in a set of cancer samples under

60 a mutational classification ${ }^{2}$, is approximated by the product of two low-rank matrices, $\boldsymbol{S}$ and $\boldsymbol{A}$.

61 The matrix $\boldsymbol{S}$ reflects the set of mutational signatures while the matrix $\boldsymbol{A}$ encompasses the

62 activities of the signatures; an activity corresponds to the number of mutations contributed by a

63 signature in a cancer sample. Algorithmically, de novo extraction of mutational signatures has

64 relied on nonnegative matrix factorization $(\mathrm{NMF})^{3}$ or on approaches mathematically analogous

65 to $\mathrm{NMF}^{4-6}$. The main advantage of NMF over other factorization approaches is its ability to yield

66 nonnegative factors that are part of the original data, thus, allowing interpretation of the

67 identified nonnegative factors ${ }^{3}$. Biologically, mutational signatures extracted from cancer

68 genomes have been attributed to exposures to environmental carcinogens, failure of DNA repair

69 pathways, infidelity/deficiency of replicating polymerases, iatrogenic events, and others ${ }^{7-14}$.

71 Since we introduced the mathematical concept of mutational signatures ${ }^{1}$, a number of

72 computational frameworks have been developed for performing de novo extraction of mutational

73 signatures (Table 1) ${ }^{14-27}$. Notably, the majority of existing de novo extraction tools (i)

74 predominately support the simplest mutational classification, viz., SBS-96 which encompasses

75 single base substitutions with their immediate 5' and 3' sequence context ${ }^{2}$; (ii) lack automatic

76 selection for the number of mutational signatures; (iii) do not identify a robust solution leading to

77 different results following re-analysis of the same dataset; (iv) require pre-selection of a large

78 number of priors and/or hyperparameters; (v) do not decompose de novo signatures to the set of 
79 reference COSMIC signatures ${ }^{14}$. Importantly, there has been no extensive benchmark of the

80 existing tools for de novo extraction leading to uncertainty regarding their performance.

82 To address these limitations, here, we present SigProfilerExtractor - a reference tool for de novo

83 extraction of mutational signatures. SigProfilerExtractor allows analysis of all types of

84 mutational classifications, performs automatic selection of the number of signatures, yields

85 robust solutions, requires only minimum setup, and decomposes de novo extracted signatures to

86 known COSMIC signatures. A comprehensive benchmark including 3,448 unique matrix

87 decompositions with SigProfilerExtractor and thirteen other tools across a total of 33 distinct

88 scenarios reveals that SigProfilerExtractor is robust to noise and that it outperforms all other

89 computational tools for de novo extraction of mutational signatures (Supplementary Tables 1-

90 3). Applying SigProfilerExtractor to the recently published set of 2,778 whole-genome

91 sequenced cancers from the Pan-Cancer Analysis of Whole Genomes (PCAWG) project ${ }^{28}$

92 elucidates three novel signatures that were not found in the original PCAWG analysis of

93 mutational signatures ${ }^{14}$. Two of the signatures are confirmed in independent cohorts and a

94 putative etiology of tobacco-associated mutagenesis is attributed to one of these signatures. 


\section{RESULTS}

Overview of SigProfilerExtractor and its implementation

98 SigProfilerExtractor is implemented as a Python package, with an R wrapper, allowing users to

99 run it in both Python and R environments:

100 https://github.com/AlexandrovLab/SigProfilerExtractor. The tool is also extensively

101 documented including a detailed Wiki page: https://osf.io/t6j7u/wiki/home/. By default, the tool

102 requires only a single parameter - the input dataset containing the mutational catalogues of

103 interest. SigProfilerExtractor supports most used formats outputted by variant calling algorithms

104 (e.g., VCF, MAF, etc.), which are internally converted to a matrix, $\boldsymbol{M}$, by

105 SigProfilerMatrixGenerator ${ }^{2}$. SigProfilerExtractor can also be applied to a text file containing a

106 matrix, $\boldsymbol{M}$, thus supporting nonnegative matrix factorization for any custom matrix dataset. By

107 default, the tool decomposes the matrix $\boldsymbol{M}$ searching for an optimal solution for the number of

108 operative signatures, $\boldsymbol{k}$, between 1 and 40 mutational signatures (Figure 1a). For each

109 decomposition, SigProfilerExtractor performs 500 independent factorizations and, for each

110 repetition, the matrix $\boldsymbol{M}$ is first Poisson resampled and normalized and, subsequently, factorized

111 with the multiplicative update NMF algorithm ${ }^{3}$ by minimizing an objective function based on the

112 Kullback-Leibler divergence measure ${ }^{29}$ (Figure 1b). Custom partition clustering, that utilizes the

113 Hungarian algorithm ${ }^{30}$ for comparing different repetitions, is applied to the 500 factorizations to

114 identify stable solutions ${ }^{31}$ (Figure $1 \boldsymbol{b}$ ). Specifically, SigProfilerExtractor selects the centroids of

115 stable clusters as optimal solutions, thus, making these solutions resistant to fluctuations in the

116 input data and to the lack of uniqueness of NMF due to the potential existence of multiple

117 convergent stationary points in the solution ${ }^{32}$. Lastly, when applicable, the optimal set of de novo

118 signatures are matched to the set of reference COSMIC mutational signatures (Figure 1c) with 
119 any de novo signature reported as novel when it cannot be decomposed by a combination of 120 known COSMIC signatures.

\section{Framework for benchmarking tools for de novo extraction of mutational signatures}

123 To allow comprehensive benchmarking of tools for de novo extraction of mutational signatures,

124 more than 60,000 unique synthetic cancer genomes were generated with known ground-truth 125 mutational signatures (Supplementary Note 1). These synthetic data included 32 distinct 126 noiseless scenarios and one scenario with five different levels of noise. Each scenario contained

127 between 3 and 40 known signatures operative in 200 to 3,000 simulated cancer genomes

128 (Supplementary Tables 1-3). Some of the scenarios were generated up to 20 times to account

129 for variability in the simulated data. Most noiseless scenarios (20/32) were based on SBS-96

130 mutational classification; 12 scenarios based on extended mutational classifications, i.e., matrices

131 with more than 96 mutational channels, were also included (Supplementary Table 3). To avoid

132 bias in evaluating each tool's performance, three sets of SBS-96 mutational signatures were used

133 for generating the synthetic data: (i) COSMICv3 reference signatures ${ }^{14}$; (ii) SA signatures

134 previously extracted by SignatureAnalyzer ${ }^{14}$; and (iii) randomly generated signatures. For

135 presentation simplicity, scenarios were labeled based on their complexity as easy, medium, or

136 hard. Easy scenarios were generated using $\leq 5$ signatures and provide a good indication of each

137 tool's performance on approximately 7.4\% of human cancer types (e.g., pediatric brain tumors).

138 Medium scenarios contained 11 to 21 signatures and biologically reflect $15.9 \%$ of cancer types

139 (e.g., cervical cancer). Hard scenarios have more than 25 signatures and reflect $59.5 \%$ of human

140 cancer types (e.g., breast, lung, liver, etc.) as well as pan-cancer datasets. In addition to the 32 
141 noiseless scenarios, one SBS-96 scenario with five different levels of noise, ranging between $0 \%$

142 and 10\%, was included in the benchmark (Supplementary Note 1).

144 To compare the performance between different tools for de novo extraction of mutational

145 signatures, we developed a standard set of evaluation metrics (Supplementary Figure 1).

146 Specifically, each de novo extracted signature is classified as either a true positive (TP), false

147 positive $(\mathrm{FP})$, or false negative $(\mathrm{FN})$ signature. An extracted signature is considered TP if it

148 matches one of the ground-truth signatures above a cosine similarity threshold of 0.90 . In

149 contrast, a signature is classified as FP when it has a maximum cosine similarity below 0.90 with

150 all ground-truth signatures. Lastly, FN signatures are ground-truth signatures that were not

151 detected in the data. These standard metrics allow calculating each tool's precision, sensitivity,

152 and $\mathrm{F}_{1}$ score. Precision is defined as $\frac{T P}{T P+F P}$, sensitivity as $\frac{T P}{T P+F N}$, and $\mathrm{F}_{1}$ score is the harmonic

153 mean of the precision and sensitivity: $2 * \frac{\text { Precision } * \text { Sensitivity }}{\text { Precision }+ \text { Sensitivity }}$.

Benchmarking SigProfilerExtractor and thirteen other tools using SBS-96 noiseless data

156 SigProfilerExtractor and thirteen other tools (Table 1) were first applied to all noiseless

157 scenarios based on the SBS-96 mutational classification. The thirteen tools include

158 SignatureAnalyzer and SigProfiler_PCAWG, a legacy MATLAB/Python version of

159 SigProfilerExtractor, which were jointly used in the PCAWG analysis of mutational signatures

160 and the derivation of the COSMICv3 set of reference mutational signatures ${ }^{14}$. Except for

161 MutSignatures which can only decompose a matrix for a fix number of signatures, all other tools

162 were applied to each scenario by using their suggested methods for selecting the number of

163 operative signatures. Apart from SignatureAnalyzer which lacks this capability, all other tools 
164 were forced to extract the known number of ground-truth signatures. Results from the suggested 165 approach reflect the expected outcome from running a tool on an unknown dataset, while results 166 from the forced approach allow understanding limitations in each tool's implementation. Our

167 evaluation reveals that most tools can successfully extract mutational signatures from easy

168 scenarios with the majority of $F_{1}$ scores between 0.90 and 1.00 (Figure $2 a$ ). This is perhaps

169 unsurprising as many of these tools used synthetic data with $\leq 5$ signatures to evaluate their

170 performance in their respective original publications ${ }^{15-17,19-26}$. In contrast, medium scenarios have

171 proven to be a challenge for most tools with only SigProfilerExtractor, SigProfiler_PCAWG, and

172 SignatureAnalyzer exhibiting $\mathrm{F}_{1}$ scores above 0.90 . All tools had worst performance for the hard

173 set of scenarios with $\mathrm{F}_{1}$ scores below 0.80 ; only SigProfilerExtractor had an $\mathrm{F}_{1}$ score of almost

1740.90 (Figure 2a).

176 To evaluate whether the type of ground-truth signatures affects the de novo extraction, we 177 compared the ratio of $\mathrm{F}_{1}$ scores $\left(\mathrm{rF}_{1}\right)$ from scenarios generated using COSMIC, $\mathrm{SA}$, or random 178 signatures (Figure $2 \boldsymbol{b})$. Most tools had similar performance $\left(\mathrm{rF}_{1} \approx 1\right)$ between COSMIC and 179 random signatures and worst performance with $\mathrm{SA}$ signatures $\left(\mathrm{rF}_{1}<1\right)$. SomaticSignatures was an 180 exception as it performed well on random signatures but had similarly suboptimal performance 181 on COSMIC and SA signatures. SigProfilerExtractor outperformed all other tools regardless of 182 whether the synthetic data were generated using COSMIC, SA, or random signatures

183 (Supplementary Table 1).

185 To examine the performance of de novo extraction between the suggested and forced selection of 186 the total number of signatures, we evaluated $\mathrm{rF}_{1}$ across all medium and hard scenarios (Figure 
$1872 \boldsymbol{2}$ ). SigProfilerExtractor exhibited almost identical $\mathrm{F}_{1}$ scores between the suggested and forced 188 selection indicating a good performance of the automatic selection algorithm. Most other tools 189 had similar $F_{1}$ scores between the suggested and forced selection albeit with more variability 190 across the different scenarios (Figure $2 c$ ). For example, MutSpec had $\mathrm{rF}_{1} \approx 1$ in both medium and 191 hard scenarios indicating that MutSpec is performing worse than SigProfilerExtractor (Figure 192 2a) not because of its algorithm for selecting the total number of signatures but likely due to its 193 implementation of the utilized numerical factorization. SigneR (hard scenarios),

194 SigProfiler_PCAWG (hard), SigMiner (medium), TensorSignatures (all), and SigFit (all) had 195 lower $\mathrm{F}_{1}$ scores for automatic solutions compared to forced solutions $\left(\mathrm{rF}_{1}<1\right)$, thus, indicating 196 that their automatic approaches for selecting the total number of signatures are not optimally 197 performing (Figure 2c). Surprisingly, EMu had higher $\mathrm{F}_{1}$ scores for automatic solutions in some 198 hard scenarios. Considering the overall performance of EMu (Figure 2a), this outcome likely 199 reflects the lack of convergence during the minimization of the EMu objective function for 200 certain number of signatures in the hard scenarios.

202 Overall, across all suggested extractions from noiseless hard scenarios reflecting $\sim 60 \%$ of human 203 cancer types, SigProfilerExtractor outperformed all other tools. SigProfilerExtractor was able to 204 identify between $10 \%$ and $37 \%$ more true positive signatures while yielding between 2.7 - and 205 16-fold less false positive signatures compared to the next seven best performing tools:

206 SigProfiler_PCAWG, SignatureAnalyzer, SigneR, MutationalPatterns, MutSpec, 207 SomaticSignatures, and SignatureTools (Figure 2 $\boldsymbol{d}$ and Supplementary Table 1). 208 209 


\section{Extended benchmarking of SigProfilerExtractor and the other seven top performing tools}

211 The reported comparisons for SBS-96 scenarios rely on a cosine similarity $\geq 0.90$ for determining

212 TP signatures and $<0.90$ for determining FP signatures. Note that a cosine similarity $\geq 0.90$ is

213 highly unlikely to happen purely by chance ( $\mathrm{p}$-value $\left.=5.90 \times 10^{-9}\right)$ as two random nonnegative

214 vectors are expected to have an average cosine similarity of 0.75 purely by chance ${ }^{33}$.

215 Importantly, SigProfilerExtractor's performance does not depend on the specific value of the 216 cosine similarity threshold (Figure $\mathbf{3 a}$ ) as the tool consistently outperforms other bioinformatics

217 approaches for almost any value of the threshold above 0.80 (p-value: 0.057 ). Cosine similarity 218 thresholds below 0.80 were not explored as extracted signature may be similar to ground-truth 219 signatures purely by chance.

220

221 Additional benchmarking was performed by generating 12 scenarios simulated using between 3

222 and 30 signatures with an extended number of mutational channels (Supplementary Note 1).

223 SigProfilerExtractor and SignatureAnalyzer are the only two tools that support analysis of

224 custom size matrices and provide GPU support (Table 1), thus, allowing analysis of data with 225 extended number of mutational channels within a reasonable timeframe. In contrast, all other 226 matrix factorization tools rely solely on CPU implementations with full runs expected to take 227 many months for each tool applied to these scenarios (Table 1). SigProfilerExtractor and

228 SignatureAnalyzer exhibited similar performance on the extended noiseless scenarios to that

229 observed on SBS-96 noiseless scenarios. Overall, SigProfilerExtractor outperformed

230 SignatureAnalyzer with average F1 scores of 0.92 and 0.85 , respectively (Supplementary Table 2312 2). 
233 To further compare SigProfilerExtractor with the other seven top performing tools, we applied

234 each tool to a dataset with 30 ground-truth SBS-96 signatures operative in 1,000 genomes and

235 random noise between $0 \%$ and $10 \%$. Analysis for each noise level was repeated 20 times to

236 account for any variability in the noise generation. SigProfilerExtractor, SomaticSignatures,

237 MutSpec, SignatureToolsLib were robust to noise with mostly unaffected performance (Figure

$238 \quad 3 b$ and Supplementary Table 3). In contrast, SigProfiler_PCAWG, SignatureAnalyzer, SigneR,

239 and MutationalPatterns were susceptible to noise (Figure 3b). For example, 2.5\% noise reduced

240 SignatureAnalyzer's $\mathrm{F}_{1}$ from 0.76 to 0.66 while $10 \%$ noise reduced its $\mathrm{F}_{1}$ to 0.07 . Similarly, $10 \%$

241 noise reduced the $F_{1}$ of SigProfiler_PCAWG from 0.76 to 0.57 , the $F_{1}$ of SigneR from 0.61 to

2420.43 , and the $F_{1}$ of MutationalPatterns from 0.60 to 0.37 . SignatureAnalyzer's reduced

243 performance on data with noise is due to its automated approach for selecting total number of

244 signatures. SignatureAnalyzer uses automatic relevance determination ${ }^{34}$ for selecting the number

245 of signatures with this number increasing from 26 (no noise; 30 ground-truth signatures) to 96

246 signatures (10\% noise; Supplementary Table 3). In contrast, SigProfiler_PCAWG, SigneR and

247 MutationalPatterns exhibit similar performance between forced and suggested solutions on data

248 with noise (Supplementary Table 3) indicating that their reduced performance is likely due to

249 the numerical implementation of their respective factorization approaches.

251 SigProfilerExtractor outperformed all other tools regardless of the levels of noise. Simulations

252 with $5 \%$ noise reflect genomics datasets with $\sim 0.95$ average sensitivity and precision of single

253 base substitutions, similar to the recently published PCAWG cohort which has 95\% sensitivity

254 (90\% confidence interval, 88-98\%) and 95\% precision (90\% confidence interval, $71-99 \%)^{28}$.

255 For simulations with 5\% noise, SigProfilerExtractor was able to identify between 20\% and 50\% 
more true positive signatures while yielding more than 5-fold less false positive signatures

257 compared to the next seven best performing tools: SigProfiler_PCAWG, SignatureAnalyzer, 258 SigneR, MutationalPatterns, MutSpec, SomaticSignatures, and SignatureTools (Figure $3 c$ and

\section{Supplementary Table 3).}

Analysis of 2,778 whole-genome sequenced human cancers with SigProfilerExtractor

262 To demonstrate its ability to yield novel biological results, SigProfilerExtractor was applied to

263 the recently published set of 2,778 whole-genome sequenced cancers ${ }^{28}$. As previously done in

264 our original PCAWG analysis of mutational signatures ${ }^{14}$, extraction of mutational signatures was

265 performed within each cancer type as well as across all samples (Supplementary Data). In

266 addition to all previously detected signatures ${ }^{14}$, our direct application of SigProfilerExtractor

267 revealed three novel mutational signatures were identified in the PCAWG dataset: SBS92,

268 SBS93, and SBS94 (Figure 4 and Supplementary Table 4).

270 Signature SBS92 was found predominately in PCAWG bladder cancers; the signature was

271 characterized by $\mathrm{T}>\mathrm{C}$ mutations with strong transcriptional strand bias consistent with damage

272 on purines for all types of single base substitutions (Figure 4a). Signature SBS92 was 9-fold

273 elevated (Figure 4d; p-value: $7.6 \times 10^{-3}$ using Wilcoxon rank sum test) in bladder cancers of ever

274 smokers compared to never-smokers in the PCAWG cohort. An almost identical signature was

275 identified by re-analyzing a recently published cohort of 88 whole-genome sequenced

276 microbiopsies of histologically normal urothelium ${ }^{35}$ with the similarity extending to both

277 trinucleotide context and transcriptional strand bias (Figure 4a; cosine similarity: 0.98; p-value <

$\left.27810^{-256}\right)$. Consistently, SBS92 was found to be 3-fold elevated in the normal urothelium of tobacco 
279 ever smokers compared to never-smokers (Figure 4d; p-value: $8.3 \times 10^{-3}$ using Wilcoxon rank 280 sum test).

282 Signature SBS93 was identified almost exclusively in PCAWG stomach cancers. SBS93 was

283 characterized by $\mathrm{T}>\mathrm{C}$ and $\mathrm{T}>\mathrm{G}$ mutations with a strand bias consistent with damage on

284 pyrimidines for TpTpA contexts (mutated base underlined; Figure $4 \boldsymbol{b}$ ). De novo extraction from

285 the Mutographs cohort of 552 whole-genome sequenced esophageal squamous cell carcinomas ${ }^{36}$,

286 a cancer type not included in the PCAWG dataset ${ }^{28}$, identified an analogous mutational signature

287 with the similarity extending to both trinucleotide context and transcriptional strand bias (Figure

$2884 \boldsymbol{b}$; cosine similarity: 0.88; p-value: $1.1 \times 10^{-6}$ ). Signature SBS94 was found at high levels in a

289 single colorectal PCAWG cancer with smaller contributions to another 8 colorectal cancers. The

290 pattern of SBS94 was characterized by C $>$ A mutations with a strand bias indicative of damage

291 on guanine (Figure $4 \boldsymbol{c}$ ). Validation of somatic mutations by visual inspection confirmed that

$29298 \%$ of mutations contributed by SBS94 are likely real. Signatures SBS93 and SBS94 did not

293 associate with any of the available PCAWG metadata ${ }^{28}$ and their etiologies remain unknown. 


\section{DISCUSSION}

296 The performed large-scale benchmarking demonstrates that SigProfilerExtractor outperforms

297 thirteen other tools for de novo extraction of mutational signatures for noiseless datasets as well

298 as for datasets containing matrices with different levels of random noise. Importantly,

299 SigProfilerExtractor generates almost no false positive signatures while still identifying a higher

300 number of true positive signatures when compared to any of the other tools (Figure $2 \boldsymbol{d}$ and

301 Figure $3 c$ ). De novo extraction of mutational signatures relies both on a factorization approach

302 and on a model selection algorithm for determining the total number of operative signatures

303 (Figure 1). Benchmarking with forced model selection, where tools were required to extract the

304 known number of ground-truth mutational signatures, reveals that SigProfilerExtractor's

305 factorization performs better when compared to the factorizations of other tools (Figure $2 \boldsymbol{a}$ and

306 Supplementary Tables 1-3). Similarly, benchmarking with suggested model selection, which

307 most closely matches analysis of a real dataset with unknown number of signatures, further

308 demonstrates SigProfilerExtractor's ability to reveal novel biological results (Figure $2 \boldsymbol{a}$ and

309 Supplementary Tables 1-3).

310

311 While our benchmarking evaluated thirteen additional tools, six of the thirteen tools internally

312 rely on the same computational engine. Maftools, MutationalPatterns, MutSpec,

313 SignatureToolsLib, SigMiner, and SomaticSignatures use the NMF R package ${ }^{37}$ to perform their

314 factorization (Table 1), albeit with slightly different hyperparameters and, in some cases, distinct

315 pre-processing of the input matrix. Predictably, these six tools have similar performance across

316 many of the scenarios (Supplementary Tables 1-3). SigProfiler_PCAWG and MutSignatures

317 utilize customize versions of an NMF implementation originally developed by Brunet et al. ${ }^{38}$ for 
318 analysis of gene expression data. TensorSignatures makes use of the standard factorization

319 algorithms included in TensorFlow ${ }^{39}$. SigFit uses a previously developed nonnegative

320 factorization method, viz., Stan R package ${ }^{40}$. In contrast, EMu, SignatureAnalyzer, SigneR, and

321 SigProfilerExtractor provide original implementations of their factorization algorithms (Table

322 1). EMu was originally developed and tested on small datasets (e.g., 21 breast genomes) ${ }^{15}$ and its

323 benchmarking performance is perhaps unsurprising considering the large number of synthetic

324 samples used in all scenarios. Surprisingly, the original implementations of SignatureAnalyzer

325 and SigneR were susceptible to noise, yielding high numbers of false-positive signatures (Figure

$3263 b)$.

327

328 Seven of the tools did not provide an automatic approach for selecting the total number of

329 operative signatures in a dataset (Table 1). Instead, most of these tools offered methodologies for

330 manually selecting the optimal number of signatures bringing user-dependence and arbitrariness

331 in selecting solutions. EMu, SigFit, SigMiner, SignatureAnalyzer, SigneR, TensorSignatures,

332 and SigProfilerExtractor provided capabilities for automatically selecting the total number of

333 operative signatures. EMu, TensorSignatures, SigneR select the total number of signatures using

334 Bayesian information criterion $(\mathrm{BIC})^{41}$, while SignatureAnalyzer and SigMiner utilize automatic

335 relevance determination $(\mathrm{ARD})^{34}$. SigFit's selection approach is based on the Elbow method ${ }^{42}$.

336 SigProfilerExtractor leverages a modified version of the NMFk selection approach which was

337 previously tested on more than 55,000 synthetic random matrices with pre-determined latent

338 factors and shown to outperform other model selection approaches ${ }^{43}$. Importantly, our

339 simulations demonstrate that SigProfilerExtractor's model selection is robust to noise while the

340 implemented BIC and ARD approaches are affected even by low levels of noise (Figure $3 \boldsymbol{b}$ ). 
341 In addition to outperforming thirteen other tools on simulated datasets, SigProfilerExtractor can

342 reveal additional biological results as demonstrated by identifying three novel signatures from

343 reanalysis of the PCAWG dataset. Importantly, SigProfilerExtractor identifies signature SBS92

344 (Figure 4) which is associated with tobacco smoking in whole-genome sequenced bladder

345 cancers and in whole-genome sequenced microbiopsies from normal bladder urothelium. The

346 strong transcriptional strand bias observed in SBS92 is indicative of an environmental mutagen

347 exposure that damages purines. Tobacco smoke is a complex mixture of at least 60 chemicals $^{13}$,

348 many capable of causing damage on purines. Interestingly, our and other prior analyses of exome

349 sequenced bladder cancers from The Cancer Genome Atlas (TCGA) project ${ }^{13,44}$ did not reveal

350 SBS92. Reanalysis of the set of TCGA bladder cancer exomes ${ }^{45}$ with SigProfilerExtractor was

351 also unable to detect SBS92 (Supplementary Data). We suspect that the lack of SBS92 in the

352 TCGA bladder cancers was due to the use of exome sequencing; note that SBS92 is

353 predominately found in intergenic regions (Figure 4a) with most samples expected to have less

354 than 15 mutations from SBS92 in their exomes. To confirm this hypothesis, we downsampled the

355 whole-genome sequenced bladder cancers and the whole-genome sequenced microbiopsies from

356 normal bladder urothelium to exomes. SigProfilerExtractor's analysis of these downsampled

357 genomes was unable to detect SBS92 confirming that exome sequencing is insufficient to

358 identify signature SBS92 (Supplementary Data).

359

360 In summary, here we report SigProfilerExtractor - a computational tool for de novo extraction of

361 mutational signatures. We demonstrate that SigProfilerExtractor outperforms thirteen other tools

362 by conducting the largest benchmarking of bioinformatics approaches for extracting mutational

363 signatures. Further, we apply SigProfilerExtractor to 2,778 whole-genome sequenced cancers 
364 and reveal several novel mutational signatures including a signature putatively attributed to

365 tobacco smoking mutagenesis in bladder cancer and in normal bladder epithelium.

366 


\section{ONLINE METHODS}

\section{Computational implementation of SigProfilerExtractor and its seven modules}

369 The implementation of SigProfilerExtractor can be separated into seven distinct modules which 370 are packaged together into a single bioinformatics tool. Module 1 processes the initial input data,

371 which can be provided as either a mutational catalogue containing a set of somatic mutations or a

372 mutational matrix. Module 2 is responsible for resampling and normalization of the mutational

373 matrix prior to performing nonnegative matrix factorization. Module 3 performs matrix

374 factorization using nonnegative matrix factorization with multiple replicates. Module 4 utilizes

375 custom clustering to derive consensus solutions and to perform model selection. Module 5

376 decomposes the derived set of de novo signatures to a set of previously derived COSMIC

377 signatures. Module 6 is responsible for calculating the activities of different signatures in

378 individual samples. Module 7 handles the extensive outputting and plotting of the different

379 analysis performed by SigProfilerExtractor. In principle, each of these modules allows extensive 380 customization. SigProfilerExtractor provides a seamless integration of these seven modules that 381 allows using them in an orchestrated and preconfigured manner with little input from a user.

\section{Module 1: Processing of input mutational catalogues or input mutational matrices}

384 SigProfilerExtractor deciphers mutational signatures from a mutational matrix $\boldsymbol{M}$ with $t$ rows 385 and $n$ columns; rows represent mutational channels while columns reflect individual cancer 386 samples (Figure 1a). The value of each cell in the matrix, $\boldsymbol{M}$, corresponds to the number of 387 somatic mutations from a particular mutational channel in each sample. The mutational matrix 388 can be provided as a text file with the first column containing the names of the mutational 389 channels and the first row containing the names of the examined samples. Alternatively, users 
can provide a mutational catalogue of somatic mutations in a commonly used format (e.g., VCF,

391 MAF, etc.) and this mutational catalogue will be internally converted into the appropriate

392 mutational matrix by SigProfilerMatrixGenerator ${ }^{2}$.

393

394

Module 2: Resampling of the input mutational matrix and normalizing the resampled matrix

395 SigProfilerExtractor does not factorize the original input matrix. Rather, prior to performing matrix factorization, SigProfilerExtractor performs independent Poisson resampling of the

397 original matrix for each replicate ${ }^{1}$. As such, the matrix factorized in each replicate is never the 398 same for a given value of $k$ (Figure $\mathbf{1 b})$. The resampling is performed to ensure that Poisson 399 fluctuations of the matrix do not impact the stability of the factorization results. Additional 400 normalization is performed after resampling to overcome potential skewing of the factorization 401 from any hypermutators. SigProfilerExtractor supports four standard normalization methods ${ }^{46}$ : 402 (i) Gaussian mixture model (GMM) normalization (default); (ii) 100X normalization; (iii) $\log 2$ 403 normalization; (iv) no normalization. No normalization does not perform any additional 404 transformation on the Poisson resampled matrix. In $\log 2$ normalization, the sum of each column 405 in the matrix is derived and logarithm with base 2 is calculated for each of these sums. Each cell 406 in a column of the matrix is multiplied by the $\log 2$ of the column-sum and subsequently divided 407 by the original column sum. In $100 X$ normalization, the sum of each column in the matrix is 408 derived. For each column where the sum exceeds 100 times the number of mutational channels 409 (i.e., 100 times the number of rows in the matrix), each cell in the column is multiplied by the 410100 times the number of mutational channels and subsequently divided by the original column 411 sum. This normalization ensures that no sample has a total number of mutations above 100 times 412 the number of mutational channels. GMM normalization encompasses a two-step process. The 
413 first step derives the normalization cutoff value in a data-driven manner using a Gaussian

414 mixture model (GMM). The second step normalizes the appropriate columns using the derived

415 cutoff value. The first step uses a GMM to separate the samples into two groups based on their

416 total number of mutations; the total number of mutations in a sample reflects the sum of a

417 column in the matrix. The group with larger number of samples is subsequently selected, and the

418 same process is applied iteratively until it converges. Convergence is achieved when the mean of

419 the two groups is separated by no more than four standard deviations of the larger group. A

420 cutoff value is derived as the average value plus two standard deviations from the total number

421 of somatic mutations in the last large group. If the derived cutoff value is below 100 times the

422 number of mutational channels, the cutoff value is adjusted to 100 times the number of

423 mutational channels. For each column where the sum exceeds the derived cutoff value, each cell

424 in the column is multiplied by the cutoff value and subsequently divided by the original column

425 sum. Note that no normalization is performed if the means of the first two groups are not

426 separate by at least four standard deviations. In all cases, columns with a sum of zero, reflecting,

427 genomes without any somatic mutations, are ignored to avoid division by zero.

430 Module 3: Matrix Factorization Using Nonnegative Matrix Factorization with Replicates

431 By default, SigProfilerExtractor factorizes the matrix $\boldsymbol{M}$ with different ranks searching for an 432 optimal solution between $k=1$ and $k=40$ mutational signatures. For each value of $k$, by default, 433 the tool performs 500 independent nonnegative matrix factorizations of the normalized Poisson 434 resampled input matrix. Thus, for each value of $k$, SigProfilerExtractor generates 500 distinct 435 factorizations of normalized Poisson resampled matrices resulting into 500 different matrices $\boldsymbol{S}$, 
each matrix reflecting the patterns of the de novo mutational signatures, and 500 different

437 matrices $\boldsymbol{A}$, each matrix reflecting the activities of the de novo mutational signatures (Figure $1 \boldsymbol{b}$ ).

438 To perform each of these factorizations, SigProfilerExtractor utilizes a custom implementation of

439 the multiplicative update algorithm ${ }^{3}$. Specifically, SigProfilerExtractor initializes the $\boldsymbol{S}$ and $\boldsymbol{A}$

440 matrices in the first step of the factorization using either random initial conditions (default) or

441 one of the derivatives of nonnegative double singular vector decomposition ${ }^{47}$.

442 SigProfilerExtractor provides internal support for minimizing three different objective functions

443 based on: (i) generalized Kullback-Leibler updates (default); (ii) Euclidean updates; (iii) Itakura-

444 Saito updates. By default, the tool performs all factorization using multithreading of central

445 processing units (CPUs) and provides support for factorization using graphics processing units

446 (GPUs) by leveraging PyTorch ${ }^{48}$. In all cases, by default, the implemented minimization

447 performs at least 10,000 iterations (also known as NMF updates or NMF multiplicative update

448 steps) with a maximum of 1,000,000 iterations. By default, the convergence tolerance of the

449 algorithm is set to $10^{-15}$. Note that SigProfilerExtractor allows configuring all factorization 450 parameters.

452 Module 4: Custom partition clustering and performing model selection

453 The previously described Module 3 generates a number of sets with each set containing, by

454 default, 500 different matrices $\boldsymbol{S}$, where each matrix reflects the patterns of de novo mutational

455 signatures for a particular factorization of a normalized Poisson resampled matrix. One set,

456 containing 500 different matrices $\boldsymbol{S}$, is generated for each of the interrogated total number of

457 operative signatures, $k$, with a default range for $k$ between 1 and 40 signatures. For each value of

$458 k$, Module 4 first performs custom clustering of the $\boldsymbol{S}$ matrices and, subsequently, applies a 
modified version of the NMFk model selection approach to select the optimal value of $k^{43}$

460 (Figure 1b). Specifically, for each value of $k$, the clustering is initialized with $k$ random

461 centroids. One of the $\boldsymbol{S}$ matrices is randomly chosen, and its columns matched to the most similar

462 centroids with no two columns assigned to the same cluster. The process is repeated until the

463 columns of all $\boldsymbol{S}$ matrices in the set are assigned to their respective clusters. SigProfilerExtractor

464 implements the Hungarian algorithm ${ }^{30}$ to pair consensus vectors from two matrices (i.e., cluster

465 centroids and mutational signature from a matrix $S$ ); the Hungarian algorithm maximizes the

466 total cosine similarities of all paired vectors between two matrices ${ }^{30}$. After assigning all columns

467 to a cluster, the centroids of each cluster are recalculated by evaluating the average of all

468 columns/vectors in a cluster. This process continues iteratively until the average silhouette

469 coefficient converges (i.e., its value does not change by more than $10^{-12}$ ). After convergence for a

470 given value of $k$, the centroids of the clusters are reported as consensus mutational signatures, an

471 overall reconstruction error is calculated for describing the original input matrix, $\boldsymbol{M}$, and stability

472 is calculated for each signature by computing the silhouette value ${ }^{49}$ of the cluster corresponding

473 to that signature (Figure $1 \boldsymbol{b}$ ). The silhouette value of a cluster measures the similarities of the

474 objects assigned to that cluster compared to any other cluster. Silhouette values range from -1.0

475 to +1.0 with values above zero indicating that, on average, objects have a higher similarity with

476 their own cluster compared to their nearest clusters. Note that signatures with low stability

477 correspond to a lack of uniqueness of the NMF due to Poisson resampling and/or to the potential

478 existence of multiple convergent stationary points in the NMF solution ${ }^{32}$.

480 Our custom clustering is performed for each of the interrogated total number of operative

481 signatures, $k$, with a default range for $k$ between 1 and 40 signatures. After performing clustering, 
482 for each value of $k$, one has derived: (i) the consensus set of mutational signatures; (ii) an overall

483 reconstruction error for describing the original input matrix; and (iii) stability value for each of

484 the identified consensus mutational signatures.

485

486 SigProfilerExtractor performs a solution selection based on the stability of signatures in a

487 solution and the ability of these signatures to reconstruct the original input matrix. By default,

488 SigProfilerExtractor will consider solutions stable if the signatures derived in the solution have

489 an average stability above 0.80 with no individual signature having stability below 0.20 . To

490 reduce overfitting, the tool also measures the information gained from the extracted set of

491 signatures in each solution. SigProfilerExtractor compares, using Wilcoxon rank-sum tests, the

492 reconstruction errors across all samples from the stable solution with the greatest number of

493 signatures to the reconstruction errors across all samples from stable solutions with lower

494 number of signatures. Stable solutions with lower number of signatures are compared in a

495 decreasing order to their total number of signatures with comparison stopping if the Wilcoxon

496 rank-sum test yields a $p$-value below 0.05 (i.e., reflecting that a solution does not describe the

497 original data as good as the stable solution with the greatest number of signatures). The stable

498 solution with lowest number of signatures and a Wilcoxon rank-sum test $p$-value above 0.05 is

499 selected as the optimal solution. If no solution has a Wilcoxon rank-sum test $p$-value above 0.05,

500 the stable solution with the greatest number of signatures is selected as the optimal solution. Note

501 that while SigProfilerExtractor selects an optimal solution, it outputs all the information

502 necessary to evaluate mutational signatures and their activities for all other stable and unstable

503 solutions. 
506 SigProfilerExtractor provides a module for decomposing each of the de novo extracted

507 mutational signatures to a set of previously derived signatures. By default, the tool decomposes

508 each of the signatures in the optimal solution to a set of COSMICv3 reference signatures ${ }^{14}$ with

509 support for signatures of single base substitutions (SBS), doublet base substitutions (DBS), and

510 small insertions and deletions (ID). Since the SBS COSMICv3 reference signatures were derived

511 under the SBS-96 classification ${ }^{2}$, any extended classification of single base substitutions (e.g.,

512 SBS-288 and SBS-1536) ${ }^{2}$ is first collapsed to the SBS-96 classification and, subsequently,

513 decomposed to the COSMICv3 reference signatures ${ }^{14}$. The decomposition functionality

514 leverages nonnegative least square (NNLS) algorithm ${ }^{50}$ as its main computational engine. A

515 mixture of addition and removal steps (add-remove functionality) were developed to estimate the

516 list of COSMIC signatures for a de novo signature. Specifically, for each de novo signature, a

517 COSMIC signature is iteratively added to a list of signatures used to explain the de novo

518 signature, NNLS is applied, and the signature which addition causes the greatest decrease of the

519 L2 error is selected. If this greatest decrease is more than a specific threshold (default value of

520 0.05) then the signature is included in the list of signatures used to explain the de novo signature.

521 The addition is immediately followed by a removal step. Each COSMIC signature in the list of

522 signatures used to explain the de novo signature are iteratively removed, NNLS is applied, and

523 the signature that causes the least decrease of the L2 error is selected. If this least decrease is less

524 than a specific threshold (default value of 1\%) then the signature is removed from the list of

525 signatures used to explain the de novo signature. The addition and removal steps are iterated until

526 no signatures are added or removed from the list of signatures used to explain the de novo

527 signature. Several previously implemented rules for mutational signatures are incorporated by 
528 default in the decomposition module ${ }^{14}$. Specifically, for signatures of single base substitutions:

529 (i) the list of signatures used to explain the de novo signature is initialized with clock-like

530 signatures SBS1 and SBS5;11 (ii) biologically connected signatures are included as previously

531 done in $\operatorname{Ref}^{14}$ (e.g., if SBS17a is included in the list then SBS17b is also included the list). The

532 decomposition module is highly customizable as it allows changing all default parameters as

533 well as adding additional new rules or removing existing rules for inclusion and exclusion of

534 particular signatures.

535

\section{Module 6: Evaluating activities of mutational signatures in individual samples}

537 De novo extracted and COSMIC derived signatures are refitted to individual samples using

538 nonnegative least squares (NNLS) ${ }^{50}$. Module 6 internally utilizes the add-remove functionality of

539 Module 5 with each sample in the original matrix, $\boldsymbol{M}$, being individually examined. For $d e$

540 novo mutational signatures, all de novo signatures are initially added to the list of signatures used

541 to explain the sample and a removal step with a cutoff of $2 \%$ is applied. To assign COSMIC

542 signatures in a sample, the module first derives the set of de novo signatures in that sample.

543 Decomposition to the COSMICv3 signatures using Module 5 is performed for each of the de

544 novo signatures and the identified COSMICv3 signatures are refitted using the add-remove

545 functionality with a removal and addition cutoffs set at $5 \%$. Finally, the activity matrix is

546 constructed by combining the activity vectors generated for all samples in the dataset.

$548 \quad$ Module 7: Outputting and plotting of analysis results

549 All previous modules make use of Module 7 for outputting and plotting of the generated results.

550 It should be noted that SigProfilerExtractor provides extensive output for the interrogated total 
551 number of operative signatures, $k$, with a default range of $k$ between 1 and 40 signatures. For

552 each value of $k$, SigProfilerExtractor outputs the set of operative de novo mutational signatures,

553 the activities of the operative signatures, and an extensive set of information related to individual

554 samples, individual de novo signatures, and the overall convergence of the factorization and

555 clustering. Module 7 also provides additional information when ran in debug mode. In addition

556 to outputting information, SigProfilerExtractor also generates a bouquet of plots both for each

557 value of $k$ as well as for the suggested optimal solution. SigProfilerExtractor utilizes all

558 previously implemented plots in SigProfilerPlotting ${ }^{2}$ as well as includes several newly developed 559 plots.

\section{Analysis of the genomics data from cancer and normal somatic tissues}

563 For all examined cancer and normal somatic tissues, de novo extraction of mutational signatures

564 was performed with SigProfilerExtractor with default parameters using two distinct mutational

565 classifications: SBS-96 and SBS-288. The SBS-96 mutation classification incorporates the six

566 types of single base substitutions: $\mathrm{C}>\mathrm{A}, \mathrm{C}>\mathrm{G}, \mathrm{C}>\mathrm{T}, \mathrm{T}>\mathrm{A}, \mathrm{T}>\mathrm{C}$, and $\mathrm{T}>\mathrm{G}$. Each type of single

567 base substitution is further separated into 16 subtypes determined by the four possible bases 5 ,

568 and 3' adjacent to each mutated base. The SBS-288 mutation classification extends the SBS-96

569 mutation classification by adding additional information for each of the 96 subtypes.

570 Specifically, SBS-288 incorporates whether a single base substitution is in non-

571 transcribed/intergenic DNA, on the transcribed strand of a gene, or on the untranscribed strand of 572 the gene. De novo extraction was performed separately for all examined datasets. Specifically,

573 SigProfilerExtractor was applied: (i) to all 2,778 whole-genome sequenced cancers from the Pan-

574 Cancer Analysis of Whole Genomes project ${ }^{28}$; (ii) to all samples in each of the 37 cancer types 
575 of Pan-Cancer Analysis of Whole Genomes project ${ }^{28}$ with each cancer type examined separately;

576 (iii) to all 88 whole-genome sequenced microbiopsies of histologically normal urothelium ${ }^{35}$; (iv)

577 to the complete set of bladder cancers from $\mathrm{TCGA}^{45}$; $(v)$ to exome downsampling of all bladder

578 whole-genome sequenced cancers from the Pan-Cancer Analysis of Whole Genomes project ${ }^{28}$;

579 (vi) to exome downsampling of all 88 whole-genome sequenced microbiopsies of histologically

580 normal urothelium ${ }^{35}$. In all cases, the mutational catalogue of each sample was taken from the

581 respective original publications. The results from all performed de novo extractions can be found

582 in Supplementary Data. Downsampling of a whole-genome sequenced sample to a whole-

583 exome was performed using SigProfilerMatrixGenerator ${ }^{2}$.

584

\section{Additional approaches for miscellaneous analysis}

586 Synthetic scenarios were labeled as easy, medium, and hard based on the number of operative 587 signatures in each scenario. Based on our most recent analysis of mutational signatures in 82 588 cancer types ${ }^{14}$, approximately $7.4 \%$ of human cancer types have 5 or less signatures (reflected in 589 simulations of easy scenarios), $15.9 \%$ have 11 to 21 signatures (medium scenarios), and 59.5\%

590 have 25 or more signatures (hard scenarios). Note that $17.2 \%$ of cancer types have either

591 between 5 and 10 signatures or between 22 and 24 signatures.

592

593 Cosine similarity was used to compare the profiles of different mutational signatures. P-values

594 can be attributed to cosine similarities based on a null hypothesis of uniform random distribution 595 of nonnegative vectors ${ }^{33}$. 
597 Briefly, the prevalence of somatic mutations in a whole-exome sample was calculated based on 598 the identified mutations in protein coding genes and assuming that an average whole-exome has 599 sufficient coverage of 30.0 megabase-pairs in protein coding genes. The prevalence of somatic 600 mutations in a whole-genome sample was calculated based on all identified mutations and 601 assuming that an average whole-genome has sufficient coverage of 3.00 gigabase-pairs.

602

603 All methods related to the generation of the benchmarking scenarios and the application of the 604 different tools to these scenarios can be found in Supplementary Note 1.

605 
606

607 TABLES

\begin{tabular}{|c|c|c|c|c|c|c|c|c|c|c|c|}
\hline \multirow{2}{*}{ Tool Name } & \multirow{2}{*}{ Input } & \multirow{2}{*}{ Platform } & \multicolumn{3}{|c|}{ Factorization Approach } & \multicolumn{3}{|c|}{ Selection Approach } & \multicolumn{2}{|c|}{ Supported Contexts } & \multirow{2}{*}{$\begin{array}{l}\text { COSMIC } \\
\text { Comparison }\end{array}$} \\
\hline & & & Method & $\begin{array}{c}\text { Computational } \\
\text { Engine }\end{array}$ & GPU & Manual & Automatic & $\begin{array}{l}\text { Automatic } \\
\text { Algorithm }\end{array}$ & $\begin{array}{c}\text { Mutational } \\
\text { Catalogue Support }\end{array}$ & Plotting & \\
\hline $\mathrm{EMu}^{15}$ & Matrix & $\mathrm{C}++$ & EM & $\begin{array}{c}\text { Original } \\
\text { implementation }^{15}\end{array}$ & No & Yes & $\mathrm{Yes}^{\wedge}$ & $\mathrm{BIC}^{41}$ & SBS-96 & No & No \\
\hline Maftools $^{16}$ & $\begin{array}{l}\text { Matrix } \\
\text { MAF }\end{array}$ & R-Bioconductor & NMF & NMF R package ${ }^{37}$ & No & Yes & No & - & SBS-96 & SBS-96 & 1 -to-1 \\
\hline MutationalPatterns $^{17}$ & $\begin{array}{l}\text { Matrix } \\
\text { VCF }\end{array}$ & R-Bioconductor & NMF & NMF R package ${ }^{37}$ & No & Yes & No & - & $\begin{array}{c}\text { SBS-96 } \\
\text { SBS-192 }\end{array}$ & $\begin{array}{c}\text { SBS-96 } \\
\text { SBS-192 }\end{array}$ & 1 -to-1 \\
\hline MutSignatures $^{18}$ & $\begin{array}{l}\text { VCF } \\
\text { MAF } \\
\text { Matrix }\end{array}$ & $\mathrm{R}$ & NMF & Brunet et al. ${ }^{38}$ & No & No & No & - & SBS-96 & SBS-96 & 1 -to-1 \\
\hline MutSpec $^{19}$ & $\begin{array}{l}\text { Matrix } \\
\text { VCF } \\
\text { Custom }\end{array}$ & $\begin{array}{l}\text { Galaxy } \\
\text { Perl } \\
\text { R }\end{array}$ & NMF & NMF R package ${ }^{37}$ & No & Yes & No & - & $\begin{array}{c}\text { SBS-96 } \\
\text { SBS-192 }\end{array}$ & $\begin{array}{c}\text { SBS-96 } \\
\text { SBS-192 }\end{array}$ & 1 -to-1 \\
\hline $\mathrm{SigFit}^{20}$ & Matrix & $\mathrm{R}$ & $\begin{array}{l}\text { Bayesian } \\
\text { Inference }\end{array}$ & Stan R package ${ }^{40}$ & No & Yes & $\mathrm{Yes}^{\wedge}$ & $\begin{array}{l}\text { Elbow } \\
\text { method }^{42}\end{array}$ & SBS-96 & $\begin{array}{c}\text { SBS-96 } \\
\text { SBS-192 }\end{array}$ & 1 -to-1 \\
\hline SigMiner $^{21}$ & $\begin{array}{l}\text { Matrix } \\
\text { MAF }\end{array}$ & $\mathrm{R}$ & $\begin{array}{l}\text { [automatic] } \\
\text { Bayesian } \\
\text { NMF } \\
\text { [manual] } \\
\text { NMF }\end{array}$ & $\begin{array}{c}\text { [automatic] } \\
\text { SignatureAnalyzer } \\
\text { implementation }{ }^{22} \\
\\
{[\text { manual] }} \\
\text { NMF R package }{ }^{37}\end{array}$ & No & $\mathrm{Yes}^{\wedge}$ & Yes & $\mathrm{ARD}^{34}$ & $\begin{array}{l}\text { SBS-96 } \\
\text { DBS-78 } \\
\text { ID-83 }\end{array}$ & Generic & 1 -to-1 \\
\hline SignatureAnalyzer ${ }^{22,23}$ & $\begin{array}{l}\text { Matrix } \\
\text { MAF }\end{array}$ & $\begin{array}{c}\mathrm{R}[\mathrm{CPU}]^{18} \\
\text { Python }[\mathrm{GPU}]^{19}\end{array}$ & $\begin{array}{l}\text { Bayesian } \\
\text { NMF }\end{array}$ & $\begin{array}{c}\text { Original } \\
\text { implementation }^{22,23}\end{array}$ & Yes & No & Yes & $\mathrm{ARD}^{34}$ & $\begin{array}{l}\text { SBS-96 } \\
\text { DBS-78 } \\
\text { ID-83 }\end{array}$ & $\begin{array}{l}\text { SBS-96 } \\
\text { DBS-78 } \\
\text { ID-83 }\end{array}$ & 1 -to-1 \\
\hline SignatureToolsLib ${ }^{24}$ & $\begin{array}{l}\text { Matrix } \\
\text { VCF } \\
\text { Custom }\end{array}$ & $\mathrm{R}$ & NMF & NMF R package ${ }^{37}$ & No & Yes & No & - & $\begin{array}{l}\text { SBS-96 } \\
\text { DBS-78 } \\
\text { ID-83 } \\
\text { SV-32 }\end{array}$ & $\begin{array}{l}\text { SBS-96 } \\
\text { SV-32 } \\
\text { Generic }\end{array}$ & No \\
\hline SigneR $^{25}$ & $\begin{array}{l}\text { Matrix } \\
\text { VCF }\end{array}$ & $\underset{\mathrm{C}++}{\mathrm{R}-\mathrm{Bioconductor}}$ & $\begin{array}{l}\text { Bayesian } \\
\text { NMF }\end{array}$ & $\begin{array}{c}\text { Original } \\
\text { implementation }{ }^{25}\end{array}$ & No & Yes & $\mathrm{Yes}^{\wedge}$ & $\mathrm{BIC}^{41}$ & SBS-96 & SBS-96 & No \\
\hline SigProfilerExtractor & $\begin{array}{l}\text { Matrix } \\
\text { VCF } \\
\text { MAF } \\
\text { Custom }\end{array}$ & $\begin{array}{l}\text { Python } \\
\text { R wrapper }\end{array}$ & NMF & $\begin{array}{l}\text { [current report] } \\
\text { Original } \\
\text { implementation }\end{array}$ & Yes & Yes & $\mathrm{Yes}^{\wedge}$ & $\mathrm{NMFk}^{43}$ & $\begin{array}{l}\text { SBS-96 } \\
\text { DBS-78 } \\
\text { ID-83 } \\
\text { CN-48 } \\
\text { Others }{ }^{2} \\
\text { Any }\end{array}$ & $\begin{array}{c}\text { SBS-96 } \\
\text { DBS-78 } \\
\text { ID-83 } \\
\text { CN-48 } \\
\text { SV-32 } \\
\text { Others }^{2} \\
\text { Generic }\end{array}$ & 1-to-many \\
\hline SigProfiler_PCAWG ${ }^{14}$ & $\begin{array}{l}\text { Matrix } \\
\text { VCF } \\
\text { MAF } \\
\text { Custom }\end{array}$ & $\begin{array}{l}\text { Python } \\
\text { MATLAB }\end{array}$ & NMF & Brunet et al. ${ }^{38}$ & No & Yes & No & - & $\begin{array}{l}\text { SBS-96 } \\
\text { DBS-78 } \\
\text { ID-83 } \\
\text { Others } \\
\text { Any }\end{array}$ & $\begin{array}{l}\text { SBS-96 } \\
\text { DBS-78 } \\
\text { ID-83 }\end{array}$ & No \\
\hline SomaticSignatures ${ }^{26}$ & $\begin{array}{l}\text { Matrix } \\
\text { VCF }\end{array}$ & R-Bioconductor & $\begin{array}{l}\text { NMF } \\
\text { PCA }\end{array}$ & $\begin{array}{c}\text { NMF R package }^{37} \\
\text { pcaMethods R } \\
\text { package }^{51}\end{array}$ & No & Yes & No & - & SBS-96 & SBS-96 & No \\
\hline TensorSignatures $^{27}$ & VCF & Python & NTF & TensorFlow ${ }^{39}$ & Yes & Yes & $\mathrm{Yes}^{\wedge}$ & $\mathrm{BIC}^{41}$ & Tensor & $\begin{array}{l}\text { SBS-96 } \\
\text { with strand } \\
\text { bias }\end{array}$ & No \\
\hline
\end{tabular}


609 Table 1: Overview of bioinformatics tools for de novo extraction of mutational signatures.

610 Tools are ordered alphabetically. Notations: ${ }^{\wedge}$ denotes the default approach for selecting the total

611 number of signatures when a tool supports both manual and automatic selection; 1-to-1 refers to

612 one de novo signature being matched with exactly one COSMIC signature; 1-to-many refers to

613 one de novo signature being matched with a combination of one or more COSMIC signatures.

614 Abbreviations: MAF: mutation annotation format; VCF: variant call format; EM: expectation-

615 maximization algorithm; NMF: nonnegative matrix factorization; NTF: nonnegative tensor

616 factorization; ARD: automatic relevance determination; BIC: Bayesian information criterion;

617 COSMIC: catalogue of somatic mutations in cancer; SBS: single base substitutions; DBS:

618 doublet base substitutions; ID: small insertions and deletions; SV: structural variants. 


\section{FIGURE LEGENDS}

621 Figure 1. Overview of SigProfilerExtractor. (a) SigProfilerExtractor's general workflow is

622 outlined starting from an input of somatic mutations and resulting in an output of de novo

623 mutational signatures. An example is shown for a solution with three de novo signatures.

624 Somatic mutations are first converted into a mutational matrix. Subsequently, the matrix is

625 factorized with different ranks using nonnegative matrix factorization. Model selection is applied 626 to identify the optimal factorization rank based on each solution's stability and its reconstruction

627 of the original data. (b) Schematic representation for an example decomposition with a

628 factorization rank of $k=3$ reflecting three operative mutational signatures. By default,

629 SigProfilerExtractor performs 500 independent nonnegative matrix factorizations with the matrix

$630 \boldsymbol{M}$ being Poisson resampled and normalized (denoted by ${ }^{\wedge}$ ) prior to each factorization. Partition

631 clustering of the 500 factorizations is used to evaluate the factorization stability rank, measured

632 in silhouette values; clustering can also be presented as two-dimensional projections revealing

633 more similar mutational signatures as shown for the three example signatures. The centroid of

634 the clustered solutions (denoted by -) is compared to the original matrix $\boldsymbol{M}$. (c) All identified de

635 novo signatures are matched to a combination of known COSMIC mutational signatures. An

636 example is given for de novo extracted signature SBS96B which matches a combination of

637 COSMIC signatures SBS1, SBS2, and SBS13.

638

639 Figure 2. Benchmarking of bioinformatics tools for de novo extraction of mutational

640 signatures using SBS-96 noiseless scenarios. (a) Average precision (x-axes), sensitivities (y-

641 axes), and $\mathrm{F}_{1}$ scores (harmonic mean of precision and sensitivity; red curves) are shown across

642 the three types of scenarios. Different tools are displayed using circles and triangles with 
643 different colors. Circles are used to display results for suggested model selection, which most

644 closely matches analysis of a real dataset. Triangles are used to display results for forced model

645 selection, where tools were required to extract the known total number of ground-truth

646 mutational signatures. All triangles are located on the diagonal as the forced model selection

647 results in equal numbers of false positive and false negative signatures. (b) Evaluating the effect

648 of ground-truth signatures on the de novo extraction by different tools (x-axes). Ratio of $\mathrm{F}_{1}$

649 scores (y-axes) with confidence intervals were calculated for medium complexity scenarios

650 simulated using COSMIC, SA, or random signatures. Ratio of approximately 1.00 indicates a

651 similar performance between different types of signatures. (c) Evaluating the performance of de

652 novo extraction between suggested and forced selection for different tools (x-axes). Ratio of $\mathrm{F}_{1}$

653 scores (y-axes) with confidence intervals were calculated for all medium and hard scenarios.

654 Ratio of approximately 1.00 indicates a similar performance between suggested and forced

655 model selection. (d) Summary of the performance for the top seven tools on hard SBS-96

656 noiseless scenarios with suggested model selection. $Y$-axes reflect $F_{1}$ score (left plot), sensitivity

657 (middle plot), and false discovery rate (right plot), respectively. Results from SignatureAnalyzer

658 and MutSignatures are not displayed in panels $(\boldsymbol{a}),(\boldsymbol{b})$, and $(\boldsymbol{c})$ for forced and suggested model

659 selections, respectively, as the tools do not support these types of analyses.

660

661 Figure 3. Additional evaluations of the top seven bioinformatics tools for de novo extraction

662 of mutational signatures. (a) Average $F_{1}$ scores for the top seven tools based on different

663 thresholds for cosine similarity in suggested medium and hard scenarios; thresholds for cosine

664 similarity are used for determining true positive signatures (Supplementary Figure 1). X-axes

665 reflect the cosine similarity thresholds, while the $\mathrm{Y}$-axes correspond to the average $\mathrm{F}_{1}$ scores 
corresponding to cosine similarity thresholds. (b) Precision and sensitivity of the top seven tools

667 for SBS-96 scenarios with different levels of noise. Noise levels reflect the average number of

668 somatic mutations in a cancer genome affected by additive white Gaussian noise; for example,

$6691 \%$ noise corresponds to approximately $1 \%$ of mutations in a sample being due to noise. (c)

670 Summary of the performance of the top seven tools on SBS-96 scenarios with 5\% noise. Y-axes

671 reflect $\mathrm{F}_{1}$ score (left plot), sensitivity (middle plot), and false discovery rate (right plot),

672 respectively.

673

674 Figure 4. Novel signatures identified in the PCAWG cohort of 2,778 whole-genome

675 sequenced cancers. Mutational signatures are displayed using 96-plots. Single base substitutions

676 are shown using the six subtypes of substitutions: $C>A, C>G, C>T, T>A, T>C$, and $T>G$.

677 Underneath each subtype are 16 bars reflecting the sequence contexts determined by the four

678 possible bases 5' and 3' to each mutated base. Additional information whether mutations from a

679 signature are in non-transcribed/intergenic DNA, on the transcribed strand of a gene, or on the

680 untranscribed strand of the gene is provided adjacent to the 96 plots. (a) Mutational profile of

681 signature SBS92 derived from the PCAWG cohort (top). Confirmation of the profile of signature

682 SBS92 (bottom) by analysis of an independent whole-genome sequenced set of microbiopsies of

683 histologically normal urothelium ${ }^{35}$. (b) Mutational profile of signature SBS93 derived from the

684 PCAWG cohort (top). Confirmation of the profile of signature SBS93 (bottom) by analysis of an

685 independent whole-genome sequenced set of esophageal squamous cell carcinomas ${ }^{28}$. (c)

686 Mutational profile of signature SBS94 derived from the PCAWG cohort. Signature SBS94 was

687 not identified in any additional independent cohort. (d) Bars are used to display average values

688 for numbers of somatic substitutions per megabase (Mb) attributed to signature SBS92 in bladder 
689 cancer and normal bladder urothelium. Green bars represent never-smokers, whereas blue bars

690 correspond to ever smokers. Error bars correspond to 95\% confidence intervals. Each p-value is 691 based on a Wilcoxon rank sum test.

692 


\section{SUPPLEMENTARY INFORMATION}

694 Supplementary Figure 1. Standard set of performance metrics used for benchmarking all

695 bioinformatics tools. An example demonstrating the derivation of true positive (TP), false

696 positive (FP), or false negative (FN) signatures for a tool applied to a synthetic dataset generated

697 using 6 ground truth signatures (termed, Ground Truth Signatures 1 through 6). The tool extracts

6984 signatures (termed, Extracted Signatures A through D). In this example, an extracted signature

699 is considered a true positive if it matches one of the ground-truth signatures with a cosine

700 similarity threshold of at least 0.90 .

701

702 Supplementary Table 1. Detailed performance metrics after applying each tool across all

703 SBS-96 noiseless synthetic scenarios. Performance metrics are calculated as per Supplementary

704 Figure 1. An extracted signature is considered a true positive if it matches one of the ground-

705 truth signatures with a cosine similarity threshold of at least 0.90 .

706

707 Supplementary Table 2. Detailed performance metrics after applying the seven best

708 performing tools across SBS-96 synthetic scenarios with different levels of noise.

709 Performance metrics are calculated as per Supplementary Figure 1. An extracted signature is

710 considered a true positive if it matches one of the ground-truth signatures with a cosine similarity

711 threshold of at least 0.90 .

712

713 Supplementary Table 3. Detailed performance metrics of applying SigProfilerExtractor

714 and SignatureAnalyzer to extended synthetic scenarios. Performance metrics are calculated 
715 as per Supplementary Figure 1. An extracted signature is considered a true positive if it matches

716 one of the ground-truth signatures with a cosine similarity threshold of at least 0.90 .

718 Supplementary Table 4. Profiles of three novel mutational signatures identified in the

719 PCAWG cohort of 2,778 whole-genome sequenced cancers. The profiles of the novel

720 mutational signatures are reported using the SBS-288 classification which incorporates the

721 trinucleotide context and strand information (intergenic region, untranscribed strand, or

722 transcribed strand) for each type of single base substitution. The SBS-288 classification can be

723 easily collapsed to the commonly used SBS-96 classification.

725 Supplementary Note 1. Detailed description of the performed benchmarking. The

726 supplementary note provides extensive details about each of the generated synthetic scenarios as

727 well as about applying each of the tools to these scenarios. The results from applying all tools to 728 all scenarios, including appropriate input and out files, can be found in Supplementary Data.

730 Supplementary Data

731 All results from the benchmarking with synthetic datasets, including the appropriate input used

732 to run each of the tools as well as the output generated by each of the tools, can be found at:

733 ftp://alexandrovlab-ftp.ucsd.edu/pub/publications/Islam_et_al_SigProfilerExtractor/Benchmark/.

735 All results from the de novo extraction of mutational signatures from the PCAWG dataset can be

736 found at: $\underline{\mathrm{ftp}}$ ://alexandrovlab-

737 ftp.ucsd.edu/pub/publications/Islam_et_al_SigProfilerExtractor/PCAWG_Reanalysis/. 
739 All results from the de novo extraction of mutational signatures for confirming the patterns of the

740 novel signatures for additional datasets can be found at: ftp://alexandrovlab-

741 ftp.ucsd.edu/pub/publications/Islam_et_al_SigProfilerExtractor/Confirmation_of_Novel_Signatu

742 res/.

743

744 All results from the de novo extraction of mutational signatures from downsampling of whole-

745 genome sequenced samples to whole-exomes can be found at: $\underline{\mathrm{ftp}} / / / \mathrm{alexandrovlab-}$

746 ftp.ucsd.edu/pub/publications/Islam et al SigProfilerExtractor/Downsampling of whole geno

$747 \underline{\text { mes/ }}$

748 


\section{ACKNOWLEDGEMENTS}

750 The authors would like to thank Allan Balmain (UC San Francisco) for the many useful

751 discussions as well as Ville Mustonen (University of Helsinki) and Israel Tojal Da Silva (A.C.

752 Camargo Cancer Center) for help in configuring EMu and SigneR, respectively. This work was

753 supported by Cancer Research UK Grand Challenge Award C98/A24032 (LBA, PB, and MRS),

754 Wellcome grant reference 206194 (MRS), as well as US National Institute of Health grants

755 R01MH116281-01A1 (BSA), R01ES030993-01A1 (LBA), and R01ES032547 (LBA). This

756 work was also supported by Singapore National Medical Research Council grants

757 NMRC/CIRG/1422/2015 and MOH-000032/MOHCIRG18may-0004 and the Singapore Ministry

758 of Health via the Duke-NUS Signature Research Programmes. LBA is an Abeloff V Scholar and

759 he is supported by an Alfred P. Sloan Research Fellowship. Research at UC San Diego was also

760 supported by a Packard Fellowship for Science and Engineering to LBA. AJG was funded by a

761 postdoctoral fellowship (grant nr. P2BSP3_178591). NP receives funding through the Cancer

762 Research UK Clinician Scientist Fellowship scheme and is supported by University College

763 London Cancer Institute. Research at Los Alamos National Laboratory was conducted under

764 Contract No. 89233218CNA000001 by the U.S. Department of Energy's National Nuclear

765 Security Administration and supported by Laboratory Directed Research and Development

766 (LDRD) grant 20190020DR (BSA). CDS is supported by the GEM consortium and

767 acknowledges funding for this work through a Cancer Research UK travel grant. The funders

768 had no roles in study design, data collection and analysis, decision to publish, or preparation of

769 the manuscript.

770

771 


\section{AUTHOR CONTRIBUTIONS}

774 LBA and SMAI designed both SigProfilerExtractor's methodology and the performed analyses 775 with help from NP, JZ, DJA, IM, BSA, LH, DCW, MTL, PB, MRS, and SGR. SMAI developed

776 SigProfilerExtractor with help from MV, ENB, YH, CDS, RV, and JW. All synthetic

777 benchmarking datasets were generated by YW and SGR. SMAI documented

778 SigProfilerExtractor and performed the benchmarking of all tools on synthetic data with help

779 from MDG, MB, BO, AK, and AA. Additional validations, confirmations, and applications of 780 SigProfilerExtractor to real and synthetic datasets were performed by SMAI, SM, SS, YRL, NS,

781 LR, TZ, AJG, YH, CDS, and SWB. LBA directed the overall research and wrote the manuscript 782 with help from SMAI and input from all other authors. All authors read and approved the final 783 manuscript.

\section{COMPETING INTERESTS}

$786 \mathrm{MV}$ is an employee of NVIDIA corporation. BSA and LBA are inventors of a US Patent

$78710,776,718$ for source identification by non-negative matrix factorization. All other authors declare 788 no competing interests.

\section{TOOL AVAILABILITY}

791 SigProfilerExtractor and all its modules are open source and freely available for use under the 792 permissive 2-clause BSD license. SigProfilerExtractor and its modules are implemented in 793 Python with an R wrapper package allowing users to run the tool from an R environment.

794 SigProfilerExtractor can be installed using the PyPI package manager from 
795 https://pypi.org/project/SigProfilerExtractor/ or downloaded from GitHub from

796 https://github.com/AlexandrovLab/SigProfilerExtractor. The R version of the tool can be

797 downloaded from https://github.com/AlexandrovLab/SigProfilerExtractorR. A detailed wiki

798 page including installation, usage, and explanation of result is provided at

799 https://osf.io/t6j7u/wiki/home/. SigProfilerExtractor is compatible with Windows, Linux, Unix,

800 and macOS operating systems.

801 
8031 Alexandrov, L. B., Nik-Zainal, S., Wedge, D. C., Campbell, P. J. \& Stratton, M. R.

804 Deciphering signatures of mutational processes operative in human cancer. Cell Rep 3,

8062 Bergstrom, E. N. et al. SigProfilerMatrixGenerator: a tool for visualizing and exploring patterns of small mutational events. BMC Genomics 20, 685, doi:10.1186/s12864-0196041-2 (2019).

3 Lee, D. D. \& Seung, H. S. Learning the parts of objects by non-negative matrix factorization. Nature 401, 788-791, doi:10.1038/44565 (1999).

4 Févotte, C. \& Cemgil, A. T. in 2009 17th European Signal Processing Conference. 1913-1917.

5 Dempster, A. P., Laird, N. M. \& Rubin, D. B. Maximum Likelihood from Incomplete Data Via the EM Algorithm. Journal of the Royal Statistical Society: Series B (Methodological) 39, 1-22, doi:10.1111/j.2517-6161.1977.tb01600.x (1977).

6 Suri, P. \& Roy, N. R. in 2017 3rd International Conference on Computational Intelligence \& Communication Technology (CICT). 1-5.

7 Alexandrov, L. B. Understanding the origins of human cancer. Science 350, 1175 , doi:10.1126/science.aad7363 (2015).

8 Alexandrov, L. B. et al. Signatures of mutational processes in human cancer. Nature 500, 415-421, doi:10.1038/nature12477 (2013).

9 Petljak, M. \& Alexandrov, L. B. Understanding mutagenesis through delineation of mutational signatures in human cancer. Carcinogenesis 37, 531-540, doi:10.1093/carcin/bgw055 (2016).

10 Pich, O. et al. The mutational footprints of cancer therapies. Nat Genet 51, 1732-1740, doi:10.1038/s41588-019-0525-5 (2019).

11 Alexandrov, L. B. et al. Clock-like mutational processes in human somatic cells. Nat Genet 47, 1402-1407, doi:10.1038/ng.3441 (2015).

12 Alexandrov, L. B., Nik-Zainal, S., Siu, H. C., Leung, S. Y. \& Stratton, M. R. A mutational signature in gastric cancer suggests therapeutic strategies. Nat Commun 6, 8683, doi:10.1038/ncomms9683 (2015).

13 Alexandrov, L. B. et al. Mutational signatures associated with tobacco smoking in human cancer. Science 354, 618-622, doi:10.1126/science.aag0299 (2016).

14 Alexandrov, L. B. et al. The repertoire of mutational signatures in human cancer. Nature 578, 94-101, doi:10.1038/s41586-020-1943-3 (2020).

15 Fischer, A., Illingworth, C. J., Campbell, P. J. \& Mustonen, V. EMu: probabilistic inference of mutational processes and their localization in the cancer genome. Genome Biol 14, R39, doi:10.1186/gb-2013-14-4-r39 (2013).

16 Mayakonda, A., Lin, D. C., Assenov, Y., Plass, C. \& Koeffler, H. P. Maftools: efficient and comprehensive analysis of somatic variants in cancer. Genome Res 28, 1747-1756, doi:10.1101/gr.239244.118 (2018).

17 Blokzijl, F., Janssen, R., van Boxtel, R. \& Cuppen, E. MutationalPatterns: comprehensive genome-wide analysis of mutational processes. Genome Med 10, 33, doi:10.1186/s13073-018-0539-0 (2018).

18 Fantini, D., Vidimar, V., Yu, Y., Condello, S. \& Meeks, J. MutSignatures: an R package for extraction and analysis of cancer mutational signatures. Scientific Reports 10, 18217 18217 (2020). 
19 Ardin, M. et al. MutSpec: a Galaxy toolbox for streamlined analyses of somatic mutation spectra in human and mouse cancer genomes. BMC Bioinformatics 17, 170, doi:10.1186/s12859-016-1011-z (2016).

852 Gori, K. \& Baez-Ortega, A. sigfit: flexible Bayesian inference of mutational signatures. bioRxiv, 372896, doi:10.1101/372896 (2020).

21 Wang, S. et al. Copy number signature analyses in prostate cancer reveal distinct etiologies and clinical outcomes. medRxiv, 2020.2004.2027.20082404, doi:10.1101/2020.04.27.20082404 (2020).

22 Kasar, S. et al. Whole-genome sequencing reveals activation-induced cytidine deaminase signatures during indolent chronic lymphocytic leukaemia evolution. Nat Commun 6, 8866, doi:10.1038/ncomms9866 (2015).

23 Taylor-Weiner, A. et al. Scaling computational genomics to millions of individuals with

24 Degasperi, A. et al. A practical framework and online tool for mutational signature analyses show inter-tissue variation and driver dependencies. Nat Cancer 1, 249-263, doi:10.1038/s43018-020-0027-5 (2020).

25 Rosales, R. A., Drummond, R. D., Valieris, R., Dias-Neto, E. \& da Silva, I. T. signeR: an empirical Bayesian approach to mutational signature discovery. Bioinformatics 33, 8-16, doi:10.1093/bioinformatics/btw572 (2017).

26 Gehring, J. S., Fischer, B., Lawrence, M. \& Huber, W. SomaticSignatures: inferring mutational signatures from single-nucleotide variants. Bioinformatics 31, 3673-3675, doi:10.1093/bioinformatics/btv408 (2015).

27 Vöhringer, H. \& Gerstung, M. Learning mutational signatures and their multidimensional genomic properties with TensorSignatures. bioRxiv, 850453 (2019).

28 Consortium, I. T. P.-C. A. o. W. G. Pan-cancer analysis of whole genomes. Nature 578, 82-93, doi:10.1038/s41586-020-1969-6 (2020).

29 Kullback, S. \& Leibler, R. A. On Information and Sufficiency. Ann. Math. Statist. 22, 7986, doi:10.1214/aoms/1177729694 (1951).

30 Kuhn, H. W. The Hungarian method for the assignment problem. Naval research logistics quarterly 2, 83-97 (1955).

31 Huang, K., Sidiropoulos, N. D. \& Swami, A. Non-Negative Matrix Factorization Revisited: Uniqueness and Algorithm for Symmetric Decomposition. IEEE Transactions on Signal Processing 62, 211-224, doi:10.1109/TSP.2013.2285514 (2014). Factorization. IEEE Transactions on Neural Networks 18, 1589-1596, doi:10.1109/TNN.2007.895831 (2007).

33 Bergstrom, E. N., Barnes, M., Martincorena, I. \& Alexandrov, L. B. Generating realistic null hypothesis of cancer mutational landscapes using SigProfilerSimulator. $B M C$ Bioinformatics 21, 438, doi:10.1186/s12859-020-03772-3 (2020).

34 Tan, V. Y. F. \& Févotte, C. Automatic Relevance Determination in Nonnegative Matrix Factorization with the /spl beta/-Divergence. IEEE Transactions on Pattern Analysis and Machine Intelligence 35, 1592-1605, doi:10.1109/TPAMI.2012.240 (2013).

35 Lawson, A. R. J. et al. Extensive heterogeneity in somatic mutation and selection in the human bladder. Science 370, 75-82, doi:10.1126/science.aba8347 (2020). 
89236 Moody, S. et al. Mutational signatures in esophageal squamous cell carcinoma from eight 893 countries of varying incidence. medRxiv, 2021.2004.2029.21255920, doi:10.1101/2021.04.29.21255920 (2021).

37 Gaujoux, R. \& Seoighe, C. A flexible R package for nonnegative matrix factorization.

38 Brunet, J. P., Tamayo, P., Golub, T. R. \& Mesirov, J. P. Metagenes and molecular pattern discovery using matrix factorization. Proc Natl Acad Sci U S A 101, 4164-4169, doi:10.1073/pnas.0308531101 (2004).

901

902

903 Abadi, M. et al. TensorFlow: Large-Scale Machine Learning on Heterogeneous Distributed Systems. arXiv e-prints, arXiv: 1603.04467 (2016).

40 Carpenter, B. et al. Stan: A Probabilistic Programming Language. Journal of Statistical Software 76, doi:10.18637/jss.v076.i01 (2017).

41 Schwarz, G. Estimating the Dimension of a Model. The Annals of Statistics 6, 461-464, doi:10.1214/aos/1176344136 (1978).

42 Thorndike, R. L. Who belongs in the family? Psychometrika 18, 267-276, doi:10.1007/bf02289263 (1953).

43 Benjamin, N., Raviteja, V., Miguel, A. H.-H., Svetlana, K. \& Boian, A. A neural network for determination of latent dimensionality in Nonnegative Matrix Factorization. Machine Learning: Science and Technology (2020).

$44 \mathrm{Kim}$, J. et al. Somatic ERCC2 mutations are associated with a distinct genomic signature in urothelial tumors. Nat Genet 48, 600-606, doi:10.1038/ng.3557 (2016).

45 Cancer Genome Atlas Research, N. Comprehensive molecular characterization of urothelial bladder carcinoma. Nature 507, 315-322, doi:10.1038/nature12965 (2014).

46 Shalabi, L. A. \& Shaaban, Z. in 2006 International Conference on Dependability of Computer Systems. 207-214.

47 Žitnik, M. \& Zupan, B. Nimfa: A python library for nonnegative matrix factorization. The Journal of Machine Learning Research 13, 849-853 (2012).

48 Lew, J. et al. in 2019 IEEE International Symposium on Performance Analysis of Systems and Software (ISPASS). 151-152.

49 Aranganayagi, S. \& Thangavel, K. in International Conference on Computational Intelligence and Multimedia Applications (ICCIMA 2007). 13-17 (IEEE).

50 Franc, V., Hlaváč, V. \& Navara, M. in Computer Analysis of Images and Patterns. (eds André Gagalowicz \& Wilfried Philips) 407-414 (Springer Berlin Heidelberg).

51 Stacklies, W., Redestig, H., Scholz, M., Walther, D. \& Selbig, J. pcaMethods--a bioconductor package providing PCA methods for incomplete data. Bioinformatics 23, 1164-1167, doi:10.1093/bioinformatics/btm069 (2007). 


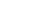

Transformation of Somatic

Mutations into a Matrix
Decomposition example for $k=3$

$\left[\begin{array}{lll}\hat{\mathbf{m}}_{11} & \cdots \\ \vdots & & \\ & & \hat{\mathbf{m}}_{t n}\end{array}\right] \approx\left[\begin{array}{lll}\mathbf{s}_{11} & \ldots \\ \vdots & & \mathbf{s}_{t 3}\end{array}\right] \times\left[\begin{array}{lll}\mathbf{a}_{11} & \ldots \\ \vdots & & \mathbf{a}_{3 n}\end{array}\right]$

$$
\hat{\mathbf{M}}_{1} \quad \mathbf{S}_{1}
$$

$\left.\mathbf{s}_{t 3}\right] \times\left[\begin{array}{l}a_{11} \\ \vdots\end{array}\right.$

$\mathbf{S}_{2}$

- $x 500$ replicates

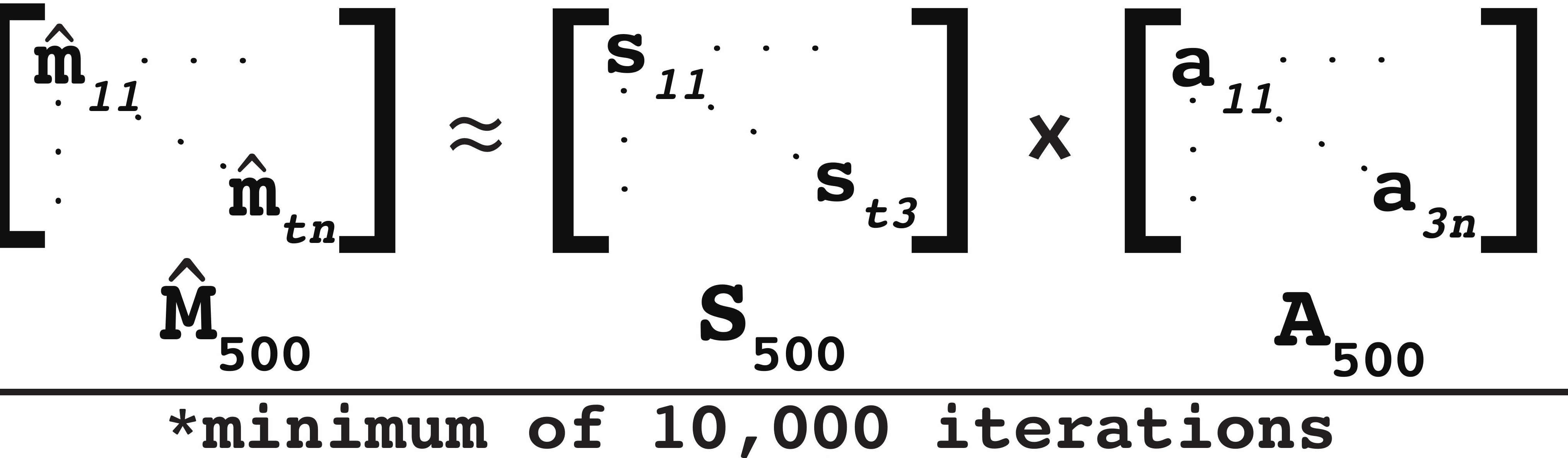

ilhouette Scores
Projection 1
Average Stability:0.98 novo extracted

signature

using COSMIC signatures

using CosMIC signatures 


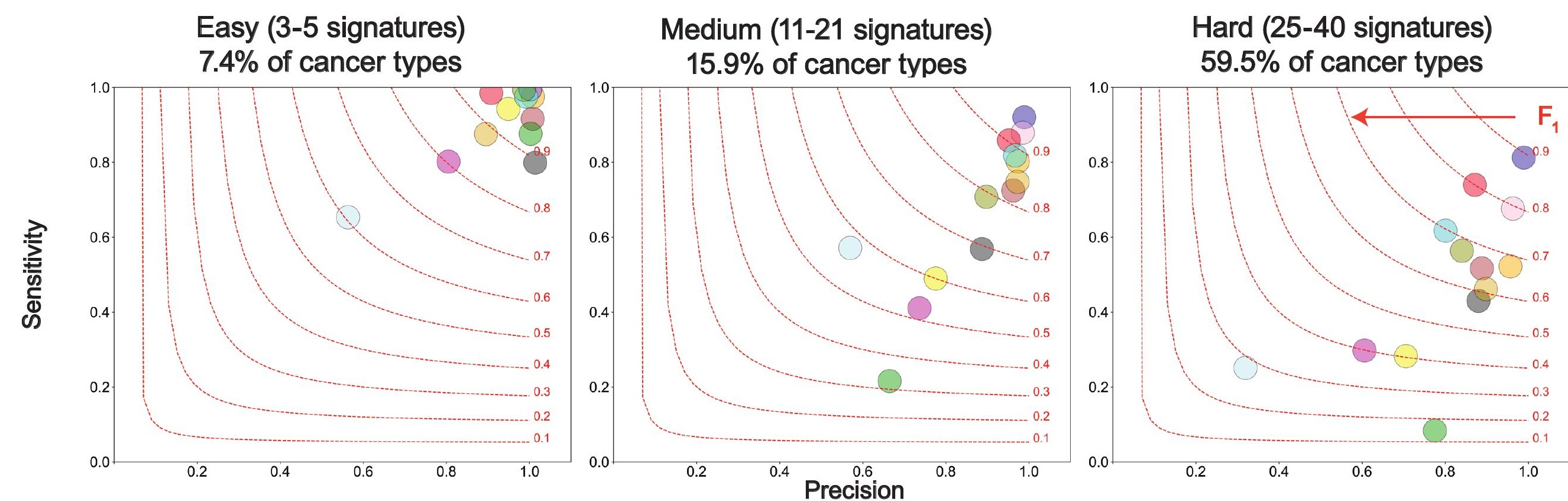

Forced

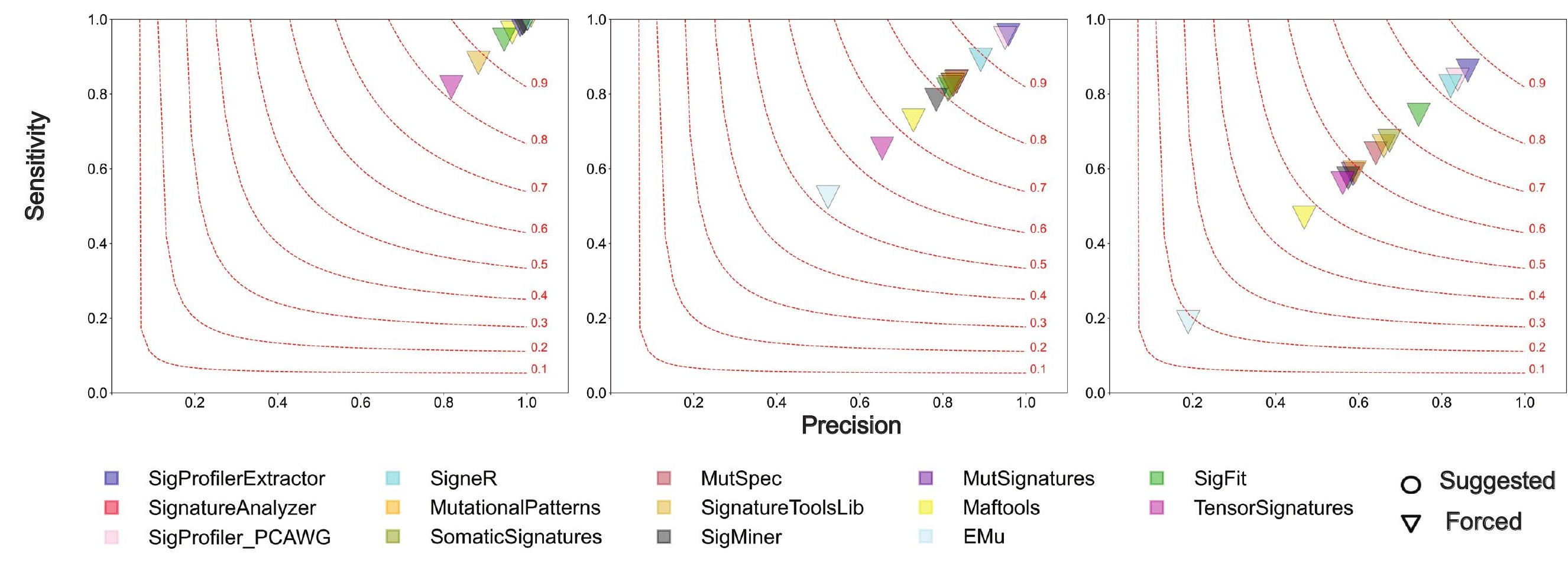

b
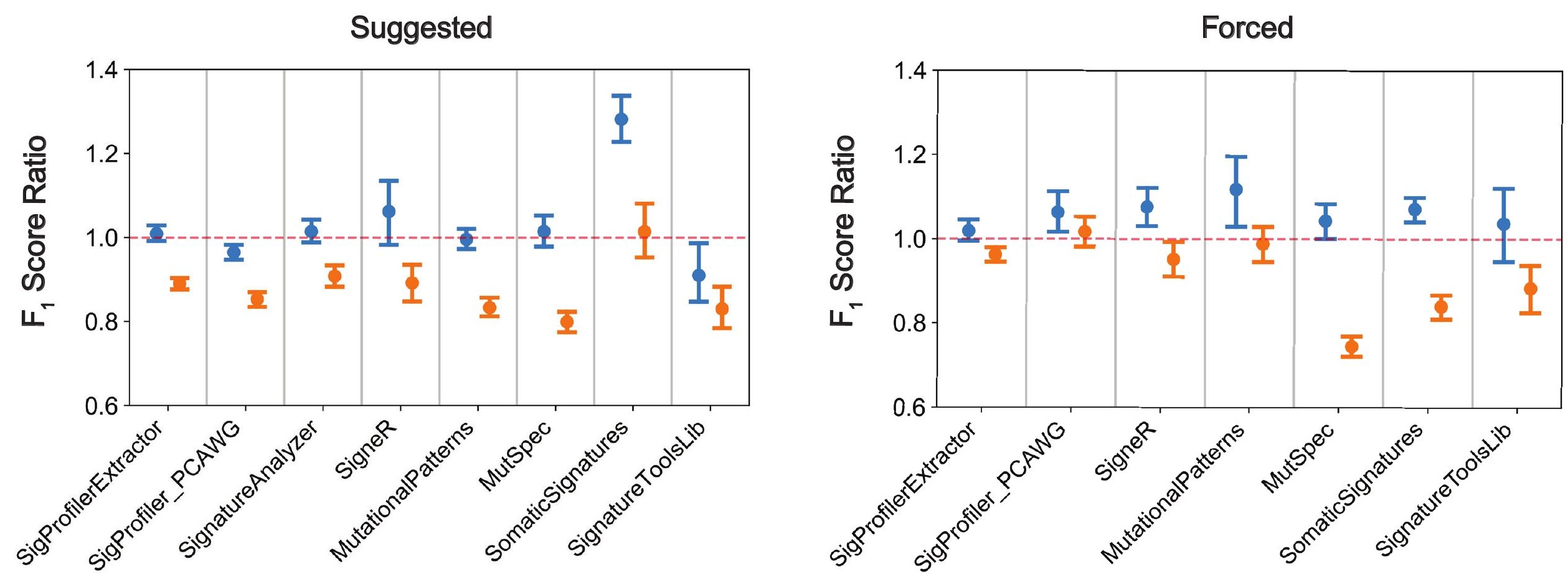

- Random signatures vs COSMIC signatures

- SA signatures vs COSMIC signatures

C

SigProfilerExtractor
SigProfiler_PCAWG
SigneR

d

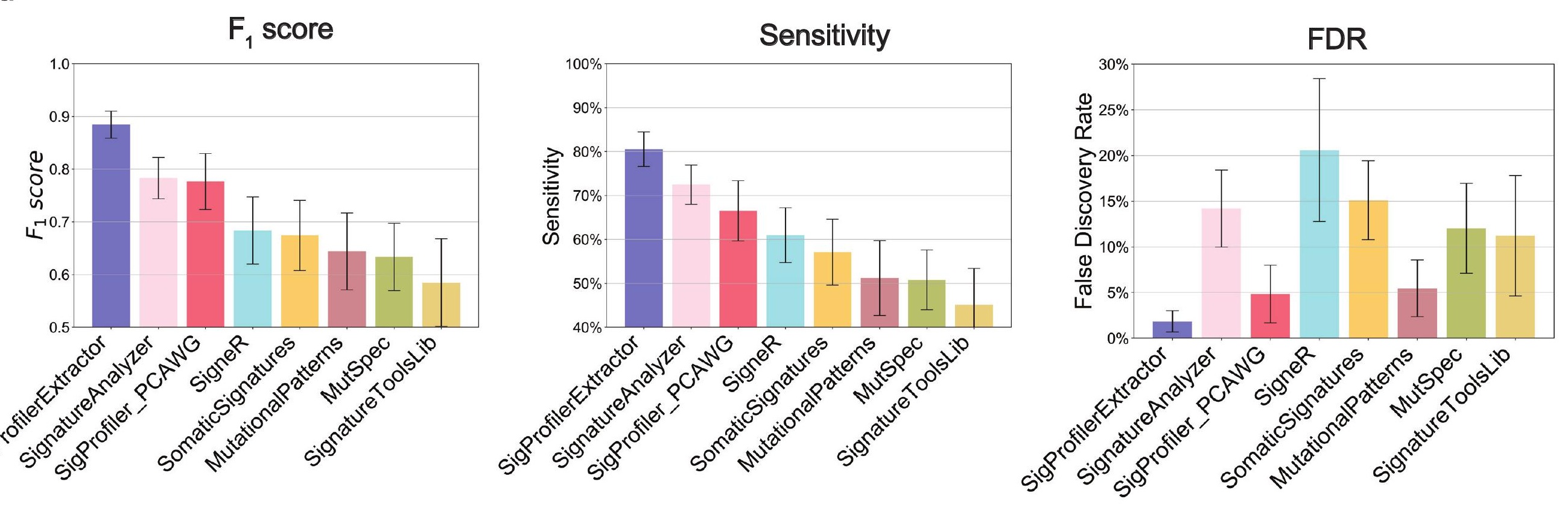



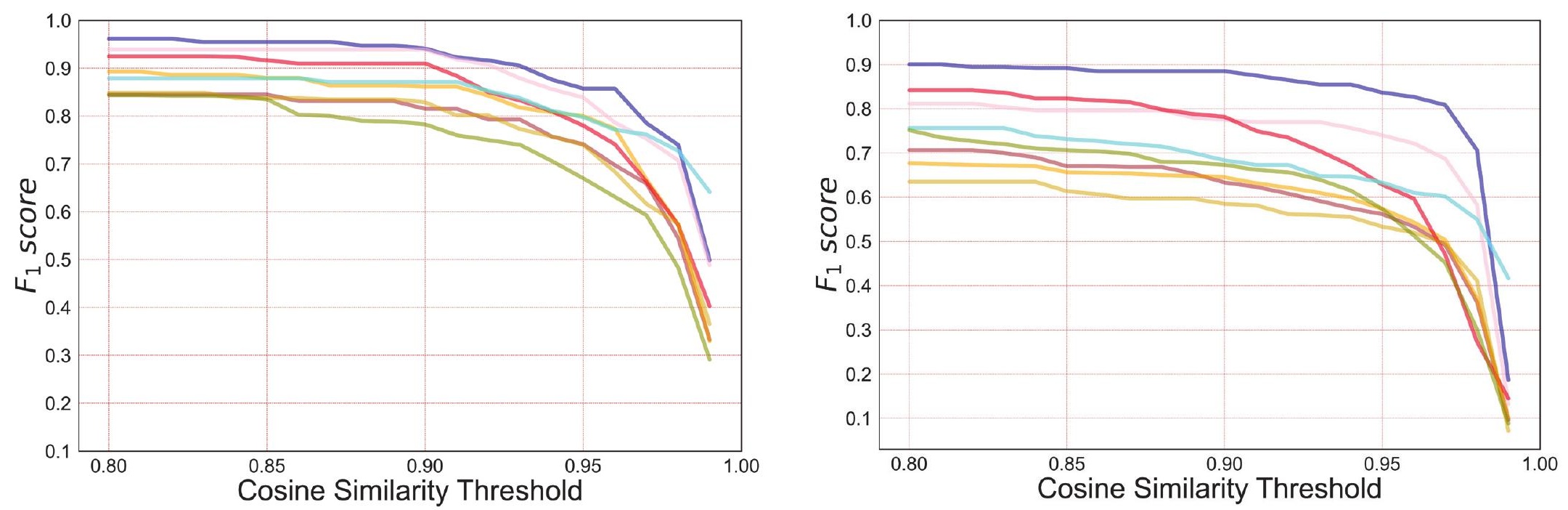

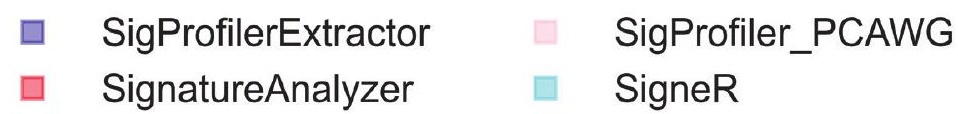

$\begin{array}{llll}\square & \text { MutationalPatterns } & \square & \text { MutSpec } \\ \square & \text { SomaticSignatures } & \square & \text { SignatureToolsLib }\end{array}$

b
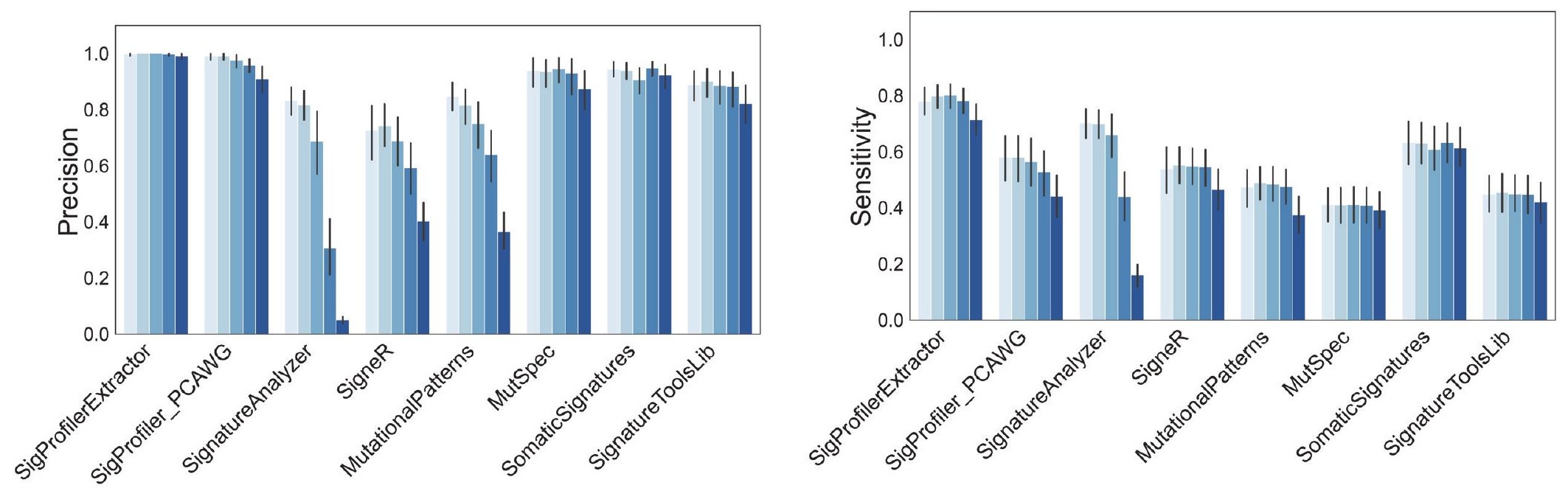

Noise \%

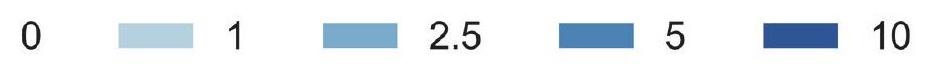

C
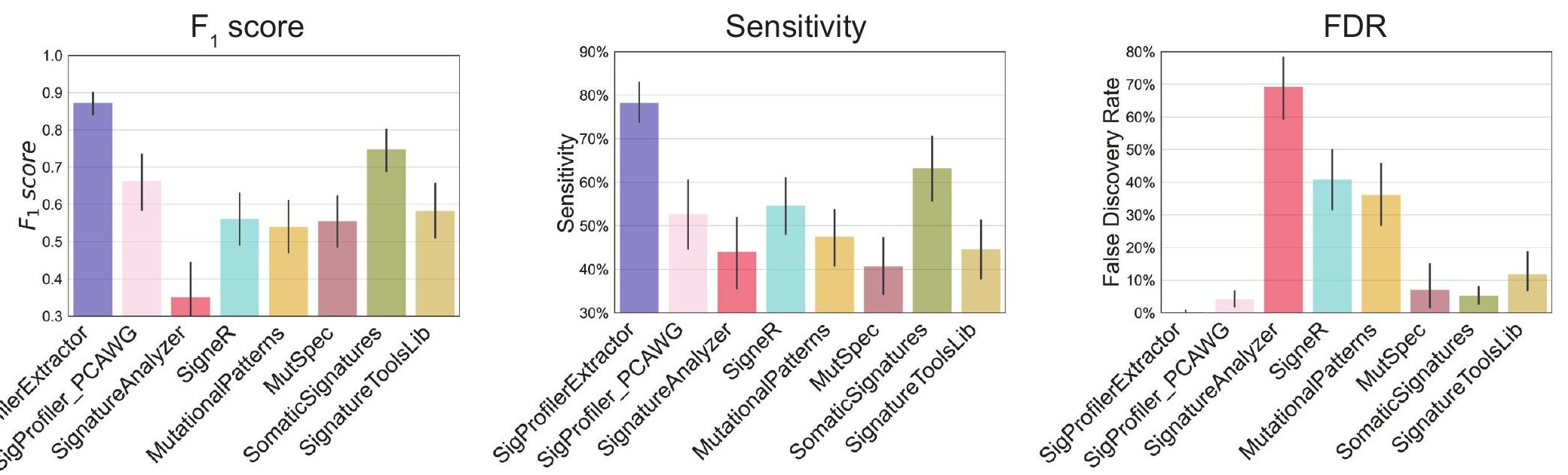
$\mathrm{T}>\mathrm{C}$

Fig. 4
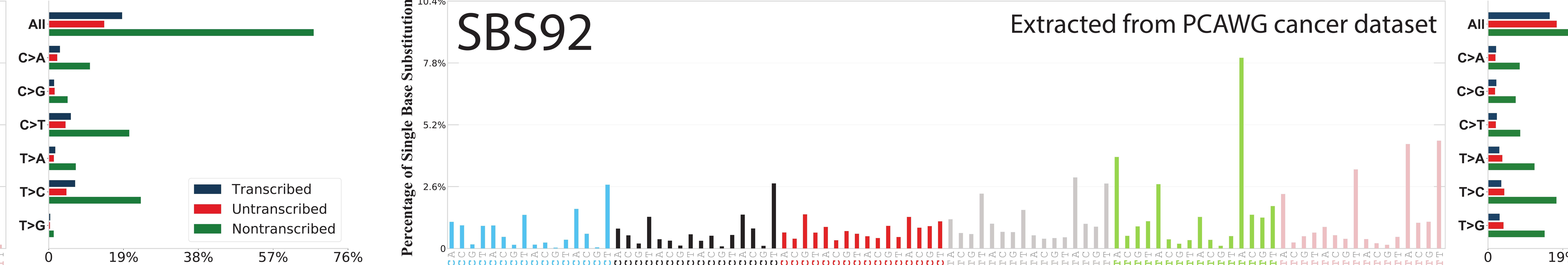

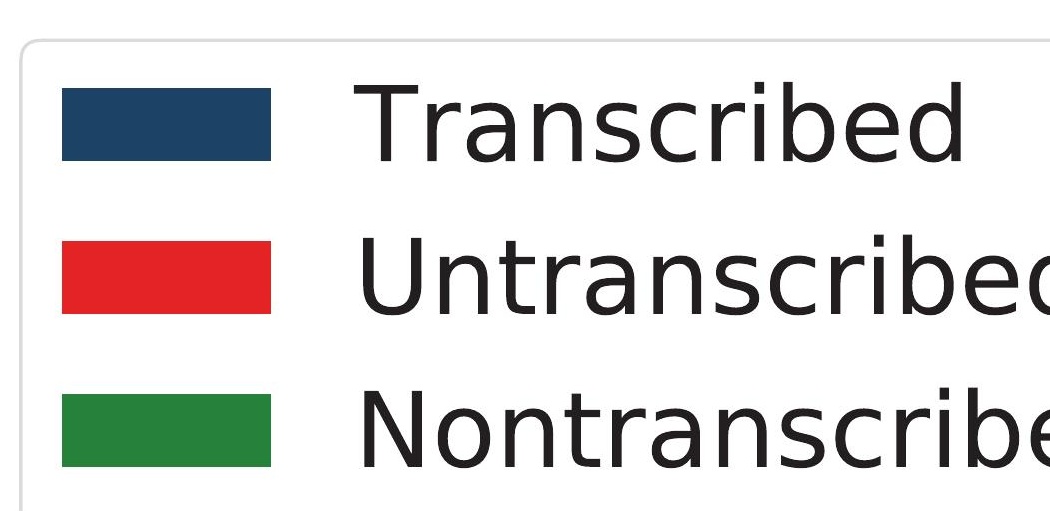

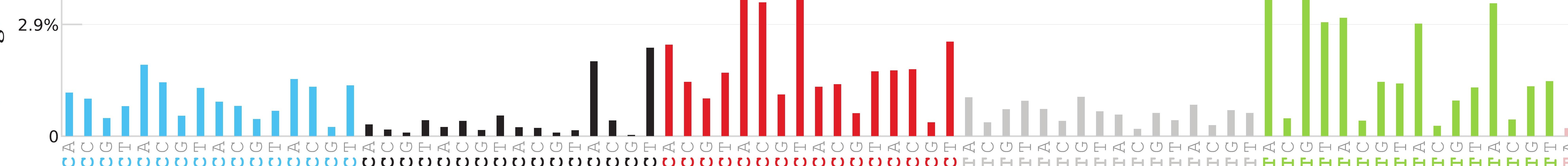

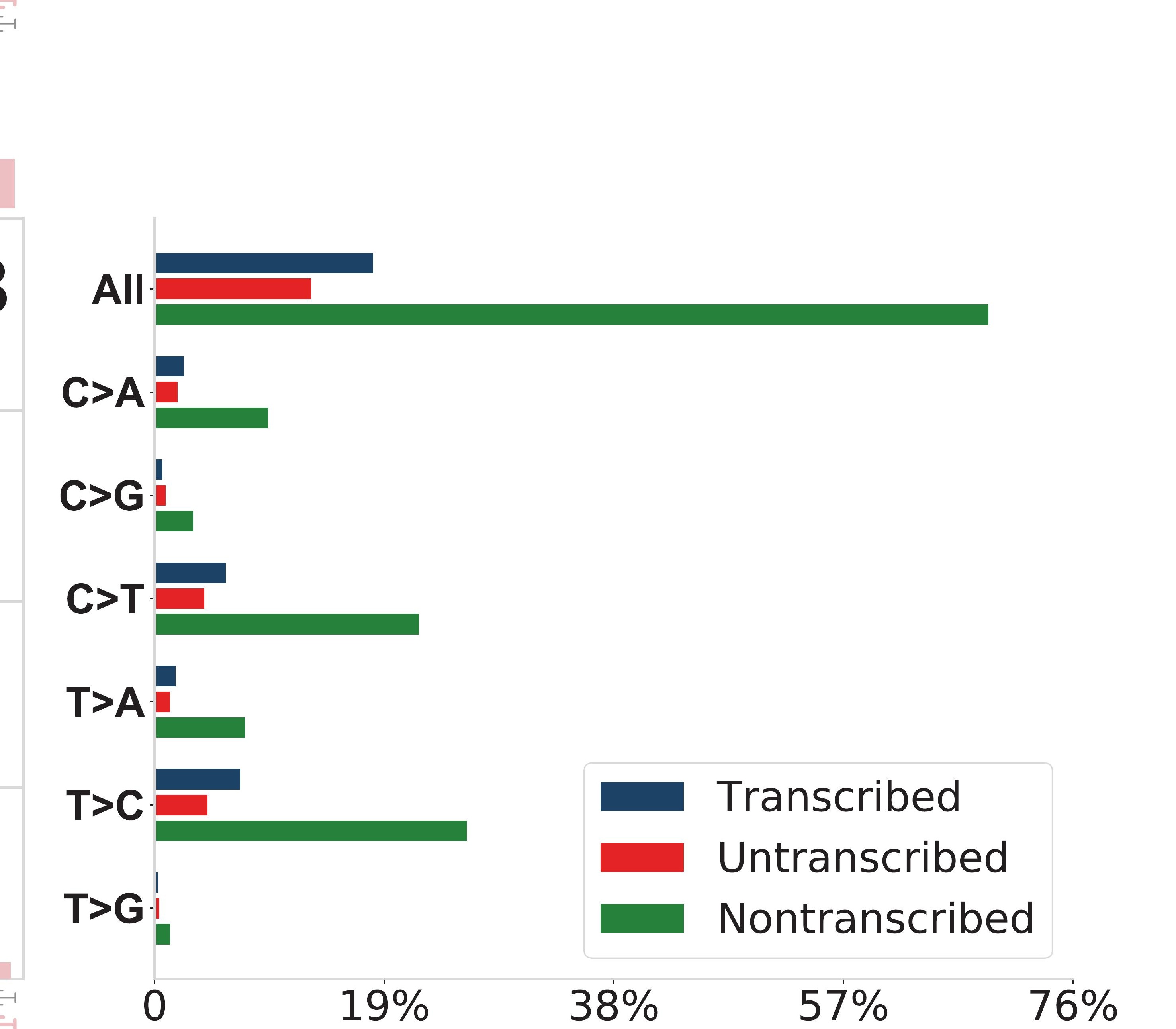

SBS91 cosine similarity: 0.98 De novo extraction from a

normal bladder urothelium cohort

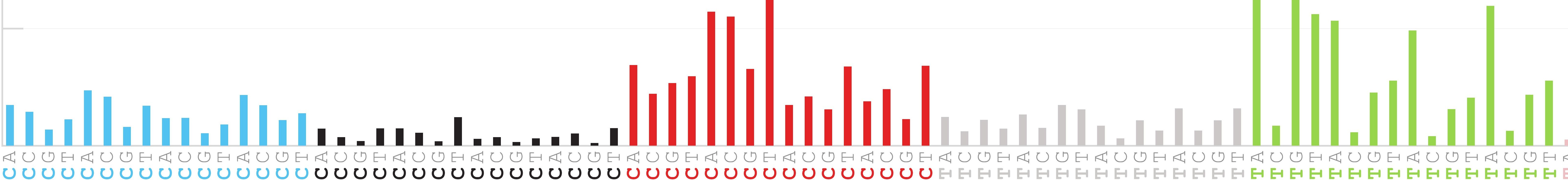

$T>G$

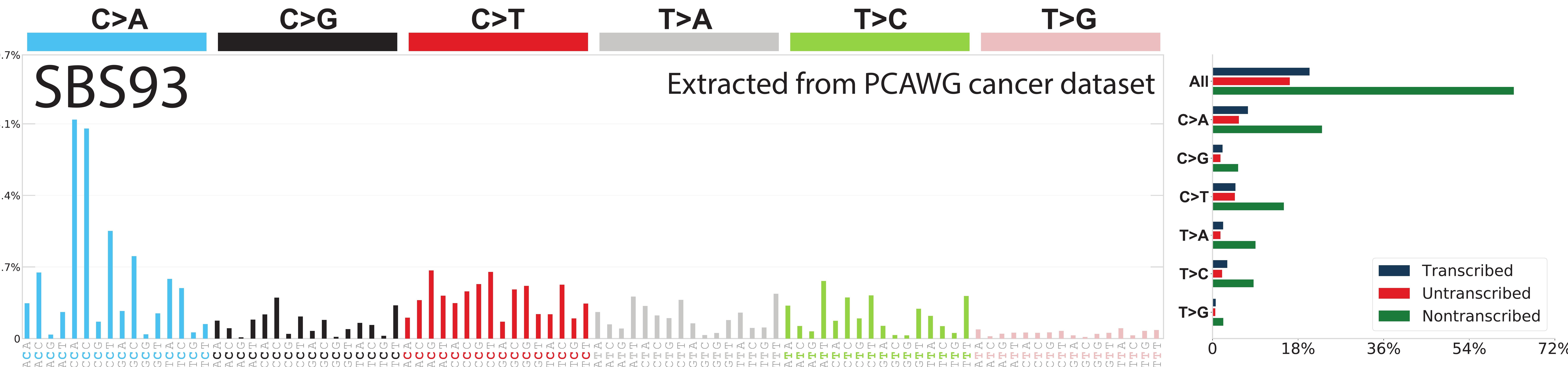

d

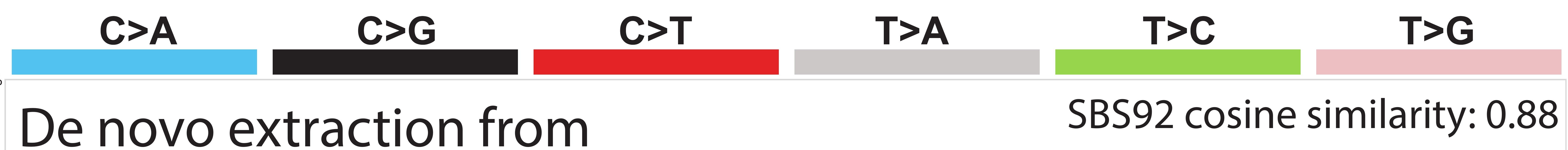

De novo extraction from

esophageal squamous cell carcinomas

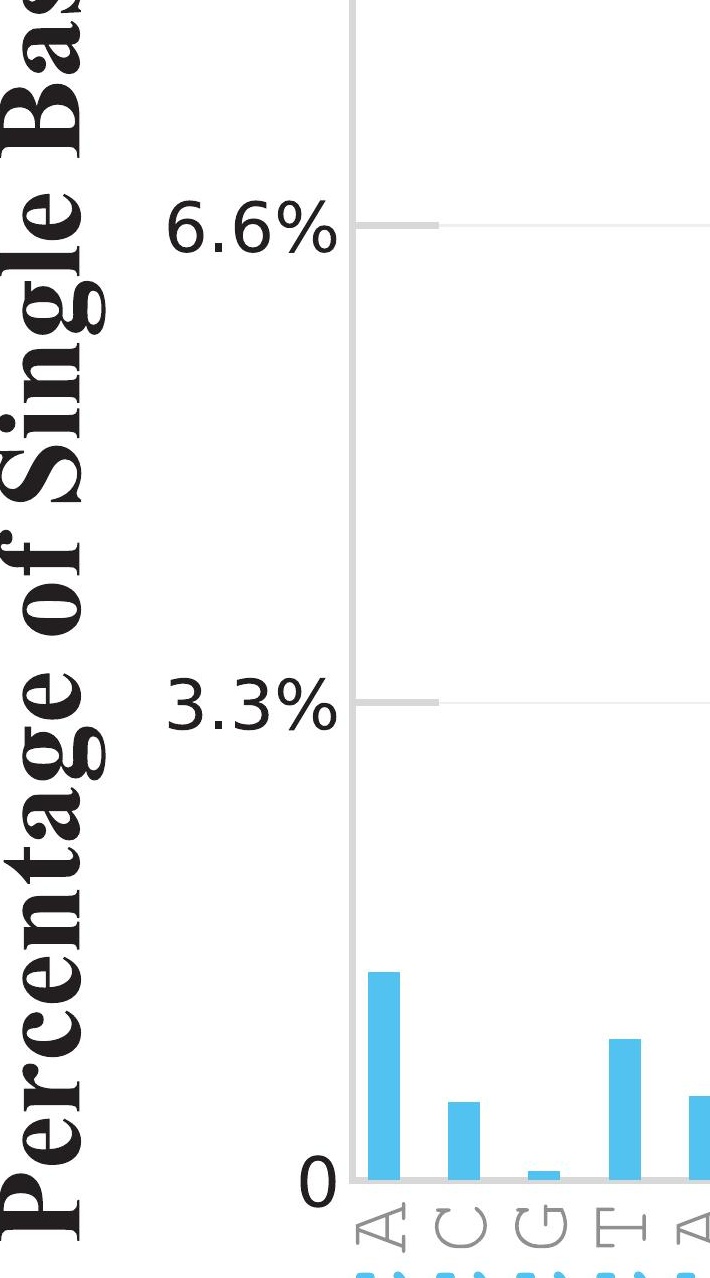

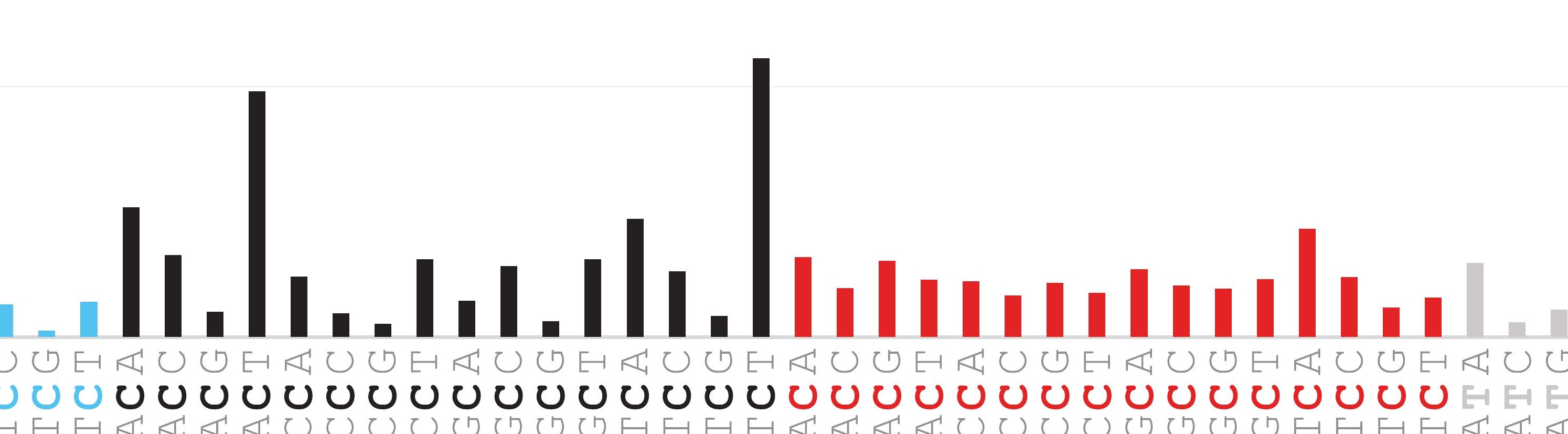
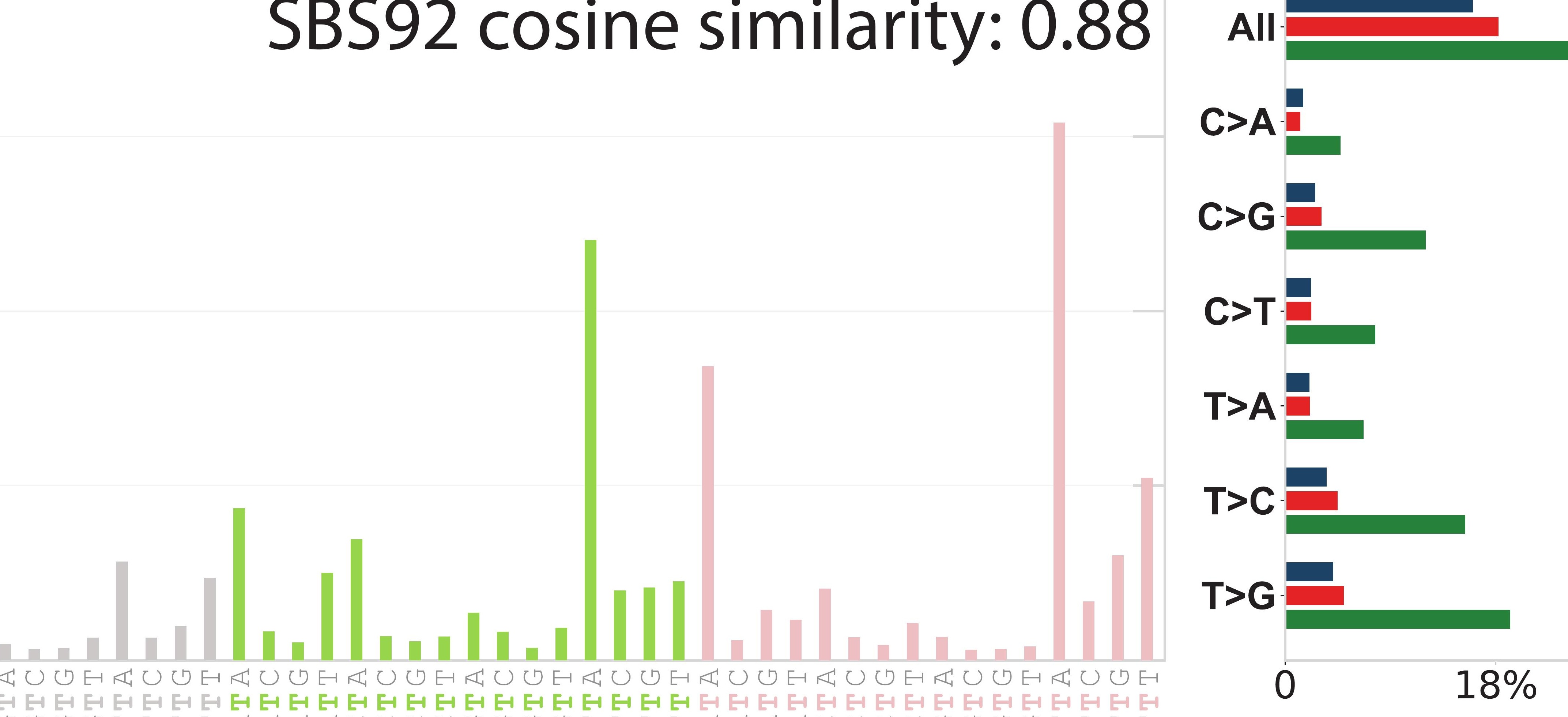

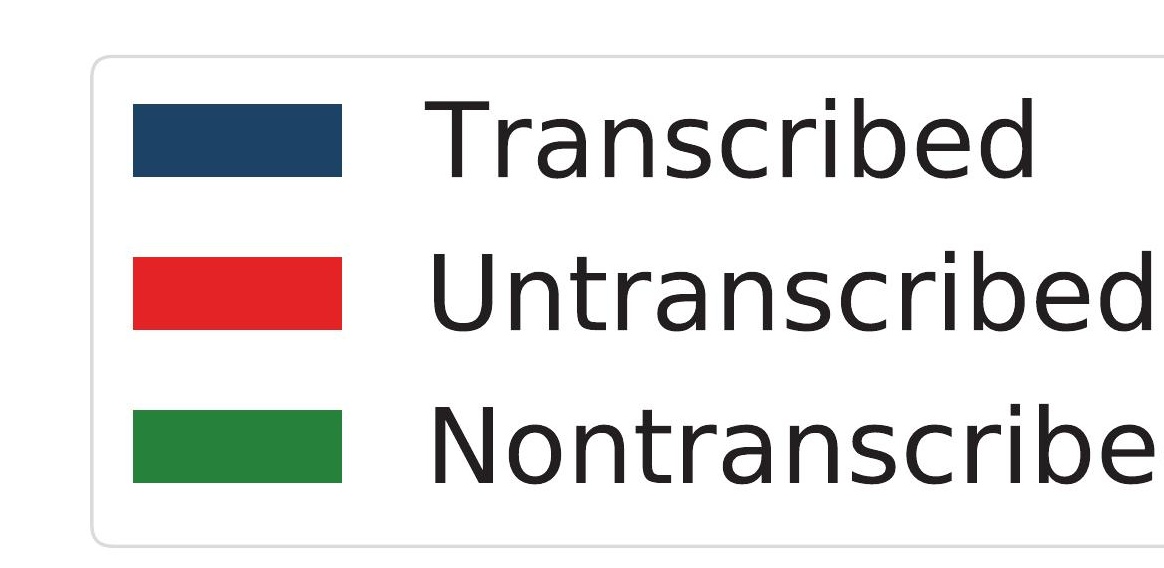
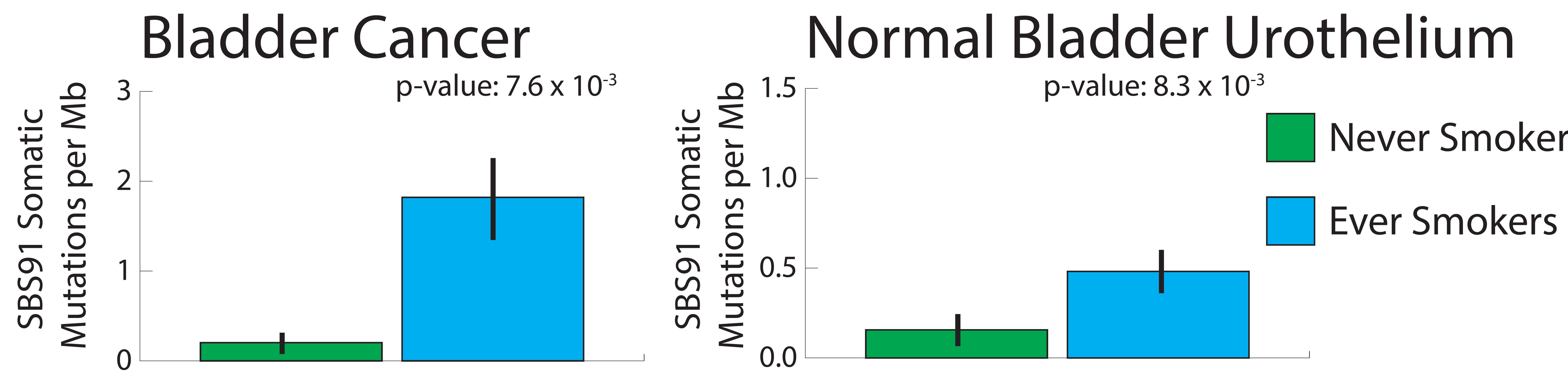